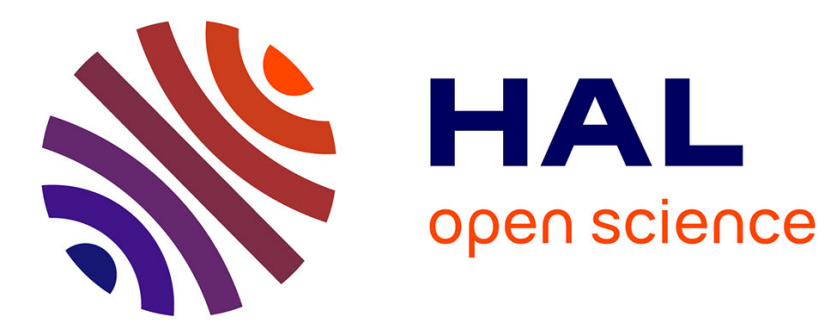

\title{
Experimental and numerical investigation of the response of a swirled flame to flow modulations in a non-adiabatic combustor
}

Adrien Chatelier, Thibault Guiberti, Renaud Mercier, Nicolas Bertier, Benoît Fiorina, Thierry Schuller

\section{To cite this version:}

Adrien Chatelier, Thibault Guiberti, Renaud Mercier, Nicolas Bertier, Benoît Fiorina, et al.. Experimental and numerical investigation of the response of a swirled flame to flow modulations in a nonadiabatic combustor. Flow, Turbulence and Combustion, 2019, 102 (4), pp.995-1023. 10.1007/s10494018-9995-2 . hal-02134631

\section{HAL Id: hal-02134631 \\ https://hal.science/hal-02134631}

Submitted on 20 May 2019

HAL is a multi-disciplinary open access archive for the deposit and dissemination of scientific research documents, whether they are published or not. The documents may come from teaching and research institutions in France or abroad, or from public or private research centers.
L'archive ouverte pluridisciplinaire HAL, est destinée au dépôt et à la diffusion de documents scientifiques de niveau recherche, publiés ou non, émanant des établissements d'enseignement et de recherche français ou étrangers, des laboratoires publics ou privés. 


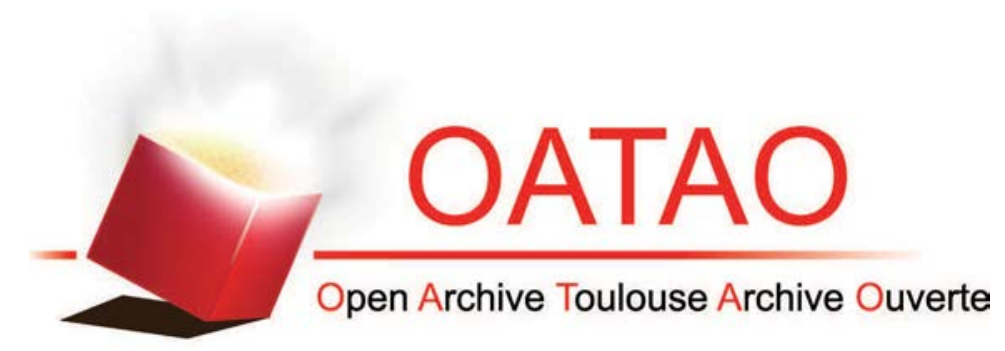

\section{Open Archive Toulouse Archive Ouverte (OATAO)}

OATAO is an open access repository that collects the work of some Toulouse researchers and makes it freely available over the web where possible.

This is an author's version published in: https://oatao.univ-toulouse.fr/23850

Official URL : https://doi.org/10.1007/s10494-018-9995-2

\section{To cite this version :}

Chatelier, Adrien and Guiberti, Thibault and Mercier, Renaud and Bertier, Nicolas and Fiorina, Benoît and Schuller, Thierry Experimental and numerical investigation of the response of a swirled flame to flow modulations in a non-adiabatic combustor. (2019) Flow, Turbulence and Combustion, 102 (4). 995-1023. ISSN 1386-6184

Any correspondence concerning this service should be sent to the repository administrator: tech-oatao@listes-diff.inp-toulouse.fr 


\title{
Experimental and Numerical Investigation of the Response of a Swirled Flame to Flow Modulations in a Non-Adiabatic Combustor
}

\author{
Adrien Chatelier ${ }^{1,2}$ (D) . Thibault Guiberti ${ }^{1,3} \cdot$ Renaud Mercier ${ }^{4}$. \\ Nicolas Bertier ${ }^{2}$. Benoît Fiorina ${ }^{1}$. Thierry Schuller ${ }^{1,5}$
}

\begin{abstract}
Turbulent combustion models for Large Eddy Simulation (LES) aims at predicting the flame dynamics. So far, they have been proven to predict correctly the mean flow and flame properties in a wide range of configurations. A way to challenge these models in unsteady situations is to test their ability to recover turbulent flames submitted to harmonic flow modulations. In this study, the Flame Transfer Function (FTF) of a $\mathrm{CH}_{4} / \mathrm{H}_{2} /$ air premixed swirled-stabilized flame submitted to harmonic flowrate modulations in a non-adiabatic combustor is compared to the response computed using the Filtered TAbulated Chemistry for LES (F-TACLES) formalism. Phase averaged analysis of the perturbed flow field and flame response reveal that the velocity field determined with Particle Image Velocimetry measurements, the heat release distribution inferred from $\mathrm{OH}^{*}$ images and the probability of presence of burnt gases deduced from OH-Planar Laser Induced Fluorescence measurements are qualitatively well reproduced by the simulations. However, noticeable differences between experiments and simulations are also observed in a narrow frequency range. A detailed close-up view of the flow field highlight differences in experimental $\mathrm{OH}^{*}$ and numerical volumetric heat release rate distributions which are at the origin of the differences observed between the numerical and experimental FTF. These differences mainly originate from the outer shear layer of the swirling jet where a residual reaction layer takes place in the simulations which is absent in the experiments. Consequences for turbulent combustion modeling are suggested by examining the evolution of the perturbed flame brush envelope along the downstream distance of the perturbed flames. It is shown that changing the grid resolution and the flame subgrid scale wrinkling factor in these regions does not alter the numerical results. It is finally concluded that the combined effects of strain rate and enthalpy defect due to heat losses are the main factors leading to small but sizable differences of the flame response to coherent structures synchronized by the acoustic forcing in the outer shear layer of the swirling flow. These small differences in flame response lead in turn to a misprediction of the FTF at specific forcing frequencies.
\end{abstract}

Adrien Chatelier

adrien.chatelier@centralesupelec.fr 
Keywords Swirling flames · Flame dynamics · Flame Transfer Function ·

Turbulent premixed combustion $\cdot$ Non-adiabatic combustion

\section{Introduction}

Prediction of combustion instabilities requires a suitable description of the turbulent flame response to incoming perturbations [1]. Experiments reveal that heat release fluctuations from swirl-stabilized turbulent flames depend on both the frequency and the level of incoming flow disturbances [2-5]. The flame response is thus often described in terms of a Flame Transfer Function (FTF) or its nonlinear extension the Flame Describing Function (FDF) [6]. Simulations of the acoustic response of swirling flames motivate research efforts from many groups [7-14] and the progress made are reviewed in $[1,15,16]$.

By explicitly computing the unsteady interactions between the largest structures of the flow field and the flame, Large Eddy Simulation (LES) is one attractive strategy to determine the FTF in practical conditions. Subgrid scale models are required to capture key quantities, such as the flame thickness or the flame wrinkling, which are not fully resolved on practical meshes $[17,18]$. As recently illustrated in a collaborative study performed in the framework of the TNF workshop [19], LES turbulent combustion models designed for turbulent premixed combustion yield good predictions of the mean statistical flame properties such as the flame front position, the temperature field, or the species mass fractions.

Studies about the ability of LES turbulent combustion strategies to retrieve flame dynamics properties and in particular its frequency response to flow modulations remain however scarce. Among the few investigations conducted, Tay-Wo-Chong et al. [20, 21] studied the impact of flame confinement and thermal boundary conditions on the FTF of a premixed swirling flame in a series of studies. They used the LES compressible code AVBP from [22] with a Dynamic Thickened Flame Model [23]. A broadband frequency perturbation method was followed to determine the FTF in the simulations [20]. Numerical results for the cold flow field were first compared to velocity measurements. The calculated heat release distribution was also compared to an image of the $\mathrm{OH}^{*}$ light distribution measured in the combustor for steady flow injection conditions. It was then shown that the broadband forcing technique allows to well reproduce the measured FTF for vanishingly small perturbation levels. No detailed comparisons of the flame motions with experiments were however carried out in these studies. An interesting conclusion is that heat losses to the walls need to be taken into account to retrieve qualitatively the flame shape observed in experiments and its FTF [21, 24-26].

The same team recently studied the combined effects of heat losses and strain rate $[27,28]$ and found as in $[29,30]$ that they strongly alter the way the flame is stabilized. These authors however did not have access to detailed experimental data for velocity and temperature fields in their combustor.

Han and Morgans [14] also chose LES to recover the FDF of a lean premixed turbulent flame stabilized in the wake of a bluff body. The incompressible solver Code_Saturne [31] with Flame Surface Density [32] and Fureby's fractal approach for flame wrinkling [33] are used in these simulations. Heat losses at the walls were not considered in this study and there is no swirl imparted to the flow. The simulations qualitatively retrieve the FDF for different perturbation levels and over all the frequency range of interest. Simulations led to underprediction of the FDF gain, but a fairly good agreement for the FDF phase lag. These authors 
also provide a qualitative comparison between the simulated flame motions and results from experiments [34] based on phase averaged analysis of the flame surface density data for different forcing frequencies. Suggestions are given for interpretation of the differences observed between experiments and simulations. A closer look of the results shown in [14] reveals that the flame dynamics is not well reproduced by the simulations close to the solid boundaries. Large differences with experiments are observed for the flame shape calculated at the flame base and flame tip. There are also no comparisons with experiments for the response of the flow field to the pulsations.

It is known that heat losses to solid boundaries modify the acoustic response of laminar flames, and in particular alter the FTF gain [35-37]. This is mainly attributed to changes of the flame response at its anchoring location. The objective of this work is to identify the issues in current turbulent combustion modeling for the simulation of the response of a swirl-stabilized flame to flow disturbances when heat losses need to be considered. The current knowledge is limited with respect to the impact of grid resolution, the choice of parameters of the subgrid scale flame wrinkling model and finally the importance of strain rate on the flame extinction for the reproduction of the flame dynamics [27, 28].

Simulations are compared in this study to measurements carried out in a labscale combustor with fully characterized flow and thermal boundary conditions. Previous experimental and numerical analyses of this setup for steady injection conditions revealed that the shape of the flame is controlled by heat losses and fuel composition [29, 30]. It was shown that the F-TACLES formalism used in these simulations allows recovering the statistical properties of the flow and the flame shape for steady flow injection conditions [30]. It is worth exploring if the same framework allows to reproduce its dynamics when the incoming flow is submitted to periodic modulations.

This article includes: (i) a comparison between experimental and numerical FTF at seven forcing frequencies and two modulation levels of velocity fluctuations, (ii) a side-by-side comparison between experiments and simulations of the phase-averaged velocity fields, heat release rate distributions and probability of presence of burnt gas distributions in an axial plane through the combustor at five regularly distributed phases in the modulation cycle and for two different forcing frequencies, and (iii) an analysis of the impact of grid resolution, subgrid scale flame wrinkling model and combined effects of strain rate and heat losses to elucidate some differences observed between the measured and simulated FTF.

The experimental and numerical setups are described in Sections 2 and 3 respectively. The procedure to determine the FTF in experiments and simulations is presented in Section 4. Comparisons between simulations and experiments for the flow and flame responses to the acoustic forcing are conducted in Section 5. These results are finally used in Section 6 to discuss the performance of the turbulent combustion model in LES.

\section{Experimental Configuration}

\subsection{The burner}

The burner sketched in Fig. 1 includes a cylindrical injection tube with a 14-mm exit diameter. A 6-mm diameter central rod installed on the burner axis helps anchoring the flame at the outlet of the injection nozzle $2 \mathrm{~mm}$ above the combustion chamber dump plane. The flow is put in rotation by a radial swirling vane located upstream of the injection tube and 


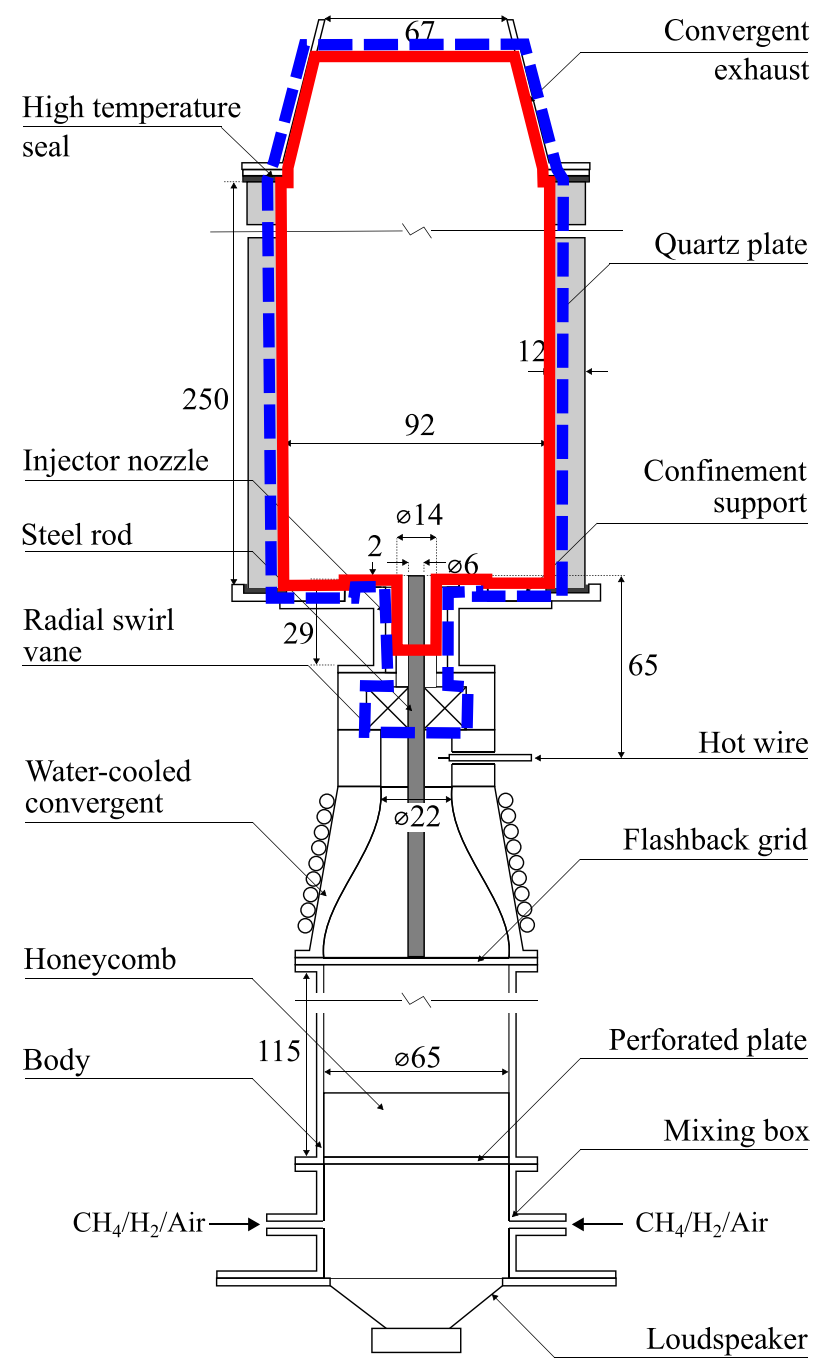

Fig. 1 Combustor geometry. The red solid line shows the computational domain CD1 for reactive flow simulations. The blue dashed lines show the computation domain CD2 for cold flow simulations. Dimensions are indicated in millimeters

yielding a swirl number of $\mathrm{S}=0.4$ that was measured by Laser Doppler Velocimetry at the burner outlet [30].

A fuel composition of $40 \% \mathrm{CH}_{4}$ and $60 \% \mathrm{H}_{2}$ in volume is selected. This fuel blend is mixed with air before being injected in the system. The combustible mixture enters the burner through a plenum and subsequently passes through a grid/honeycomb/grid arrangement before entering a water-cooled convergent nozzle to reach a nearly uniform top-hat velocity profile at the entrance of the swirler. A loudspeaker is placed at the bottom of the injection system to modulate the flow. The flame is stabilized in the combustion chamber featuring four $250 \mathrm{~mm}$ (height) $\times 92 \mathrm{~mm}$ (width) $\times 12 \mathrm{~mm}$ (thickness) quartz windows. 
The equivalence ratio of the fuel/air feeding stream is $\phi=0.7$ and the thermal power is $P=4 \mathrm{~kW}$ assuming complete combustion. In the absence of forcing, these conditions lead to a V-shaped flame stabilized by the swirling flow and the central bluff-body [29, 30].

\subsection{Diagnostics}

The axial, radial and azimuthal components of the velocity field, the flame structure and the thermal boundary conditions were fully characterized in [30] for steady flow injection conditions. Wall temperatures were measured with thermocouples in the vertical steel bars of the combustion chamber and with Laser Induced Phosphorescence (LIP) along the quartz windows and the dump plane of the combustor. Only measurements under perturbed flow conditions are presented in this study.

The FTF is measured with a hot wire (Dantec Dynamics, Mini CTA 54T30 and probe 55P11) and a photomultiplier (Hamamatsu, H5784-06). The hot wire is introduced in the stream of fresh reactants $65 \mathrm{~mm}$ upstream of the injector outlet as indicated in Fig. 1. In this cross-section, upstream of the radial swirling vane, the flow is axial, laminar with a top hat velocity profile. The photomultiplier is equipped with a $10 \mathrm{~nm}$ bandpass filter centered at $307 \mathrm{~nm}$ (CVI, F10-307.1-4-50.0M) and is used to detect the $\mathrm{OH}^{*}$ global emission from the flame. The $\mathrm{OH}^{*}$ signal is not linearly related to the heat release rate as there are also dependencies on equivalence ratio, strain and the combustion regime. It is however an acceptable indicator for lean premixed conditions as the $\mathrm{OH}^{*}$ species profiles is not affected by local fuel stratification. For this reason, this quantity has been used in the past in many analysis of the dynamics of lean premixed flames as for example in [3]. A discussion about the validity of this assumption can be made in configurations with stratified equivalence ratio, strain or combustion regime effects, but it is assumed here that these effects do no affect the conclusion on the fluctuations of $\mathrm{OH}^{*}$ global emission.

The flame response to to flow modulations is also analyzed by imaging the spatial distribution of $\mathrm{OH}^{*}$ emission with a $1024 \times 1024$ ICCD camera (Princeton Instruments, PI-MAX 4) equipped with a UV lens (UV-NIKKOR $105 \mathrm{~mm}$ ) and a 10-nm bandpass filter centered at $310 \mathrm{~nm}$ (Asahi spectra CO., ZBPA310). The distribution of hot burnt gases is inferred from Laser Induced Fluorescence of the OH radical (OH-PLIF) in a light sheet through the axial plane of the burner. The OH-PLIF setup is standard and is described in [29, 30]. For a given forcing frequency, $\mathrm{OH}^{*}$ and $\mathrm{OH}-\mathrm{PLIF}$ images are phase averaged over more than 100 snapshots to get a statistically converged information at five regularly distributed phases within a modulation period. These data are used to analyze the flame motion.

Particle Image Velocimetry (PIV) is also used to characterize the modulated flowfield in reacting conditions and analyze the dynamics of large coherent structures in the shear layers of the swirled jet during a modulation period. The PIV setup and flow seeding are fully described in $[29,30]$.

\section{Numerical Setup}

\subsection{Numerical setup and combustion modeling}

Simulations are conducted with the incompressible, unstructured finite volume YALES2 flow solver [38]. Numerical schemes are fourth order in space discretization and time integration. 
Two computational domains are defined in Fig. 1. The first one (CD1, red solid line in Fig. 1) does not include the swirler and is used for reactive simulations. The inlet of CD1 is located $15 \mathrm{~mm}$ downstream of the swirler outlet. The mesh includes 49 millions of tetrahedral elements. The characteristic mesh size in the flame region is $0.3 \mathrm{~mm}$. The second computational domain (CD2, blue dashed lines in Fig. 1), meshed with 50 millions of tetrahedral elements, includes the swirler and is dedicated only to cold flow simulations. As explained in [30], this second numerical domain is used to identify the inlet boundary conditions of the reactive flow simulations.

The SIGMA model is retained to model the subgrid scale turbulent stresses [39]. Reactive numerical simulations are performed with the F-TACLES turbulent combustion model based on the tabulation of filtered 1-D premixed flames [40] extended to account for heat losses by [41]. The heat losses are taken into account more specifically by introducing the enthalpy defect $\Delta h=h_{a d}-h_{h l}$, where $h_{a d}$ is the chemical plus sensible enthalpy in the fresh gases and $h_{h l}$ the chemical plus sensible enthalpy with heat losses at the wall. The enthalpy $h_{h l}$ is transported as a passive scalar with boundary conditions linked to the imposed temperature boundary conditions. More details on this model can be found in [41].

Chemical look-up tables are computed with the REGATH thermochemistry package [42] using the detailed chemical scheme from [43], which includes 29 species and 141 reactions. The chemistry tabulation procedure captures the impact of differential diffusion and heat losses on the flame consumption speed by using the methodology developed in [41]. A filter width of $\Delta=8 \delta_{0}$, where $\delta_{0}=0.44 \mathrm{~mm}$ is the laminar flame thickness of the $\mathrm{CH}_{4} / \mathrm{H}_{2} /$ air flame, is chosen to build the F-TACLES table. This flame filter width ensures a proper resolution of the flame front propagation without introducing numerical artifacts [40]. The subgrid scale model for the flame wrinkling is based on the formulation of [44], where the $\beta$ parameter is set to 0.5 , a value that allowed to capture the correct average flame position in [30].

The walls are considered as isothermal with a temperature distribution that is interpolated and extrapolated using the measurements made along the metallic and quartz wall surfaces. More details on these measurements and the way the thermal boundary conditions are imposed in the simulations are presented in [30]. A logarithmic wall law is used in the simulations to recover the proper friction at the wall despite not being resolved by the mesh.

\subsection{Results without forcing}

Simulations without external forcing were carried out in [30]. Figure 2 indicates that the V flame shape is correctly reproduced by the LES. The flame length is overestimated by only a few millimeters, but the zone of maximum heat release spreads over a larger region in the simulation than the $\mathrm{OH}^{*}$ signal in the experiment. Differences are also observed in the outer shear layer of the swirling jet where the numerical results exhibit a weak but perceptible reactive layer. There is however no $\mathrm{OH}^{*}$ light detected in the experiments at these locations for $6 \mathrm{~mm} \leq|x| \leq 11 \mathrm{~mm}$ and $5 \mathrm{~mm} \leq z \leq 18 \mathrm{~mm}$. One refers to [30] for a more detailed discussion on this aspect.

\subsection{Flow forcing}

A pulsed cold flow simulation is performed on CD2 to determine the inlet boundary conditions for the reactive simulations on CD1. The inlet flow velocity is submitted to an harmonic oscillation at a frequency $f=350 \mathrm{~Hz}$ and a modulation level $u_{z}^{\prime} / \bar{u}_{z}=0.17$, 

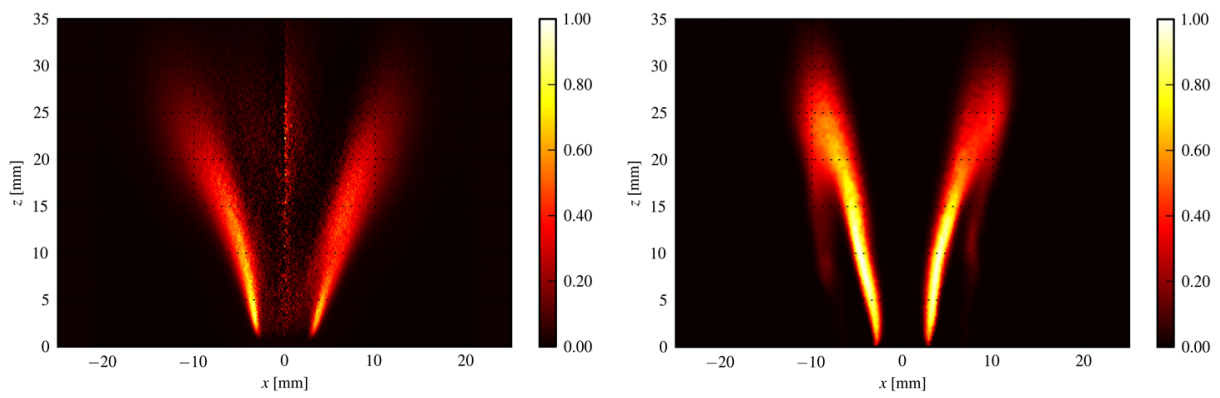

Fig. 2 Unforced case comparison between experiments (Abel deconvoluted $\mathrm{OH}^{*}$ chemiluminescence signal) at the left and simulations (heat release rate) at the right. The signals are normalized by their maximum value. Adapted from [30]

where $\bar{u}_{z}$ is the local axial velocity in the 22-mm diameter injection channel (with the central insert) and $u_{z}^{\prime}$ denotes the fluctuation amplitude. The signals for the axial and azimuthal velocity components are extracted from planes at $z=-35 \mathrm{~mm}$ corresponding to the exit of the swirler and $z=-20 \mathrm{~mm}$ corresponding to the reactive simulations inlet as shown in Fig. 3.

Phase averaged results to eliminate effects of turbulence are shown in Fig. 4. The axial velocity at the exit of the swirler (solid blue line in Fig. 4) and at the inlet of the numerical domain CD1 (red dashed lines in Fig. 4) separated by a distance $d=15 \mathrm{~mm}$ are in phase. This is not the case for the azimuthal component of velocity that features a phase shift $\Delta \varphi=2.19$ rad due to the presence of the swirler, which generates a vorticity wave as a response to the axial pulsation [2,3]. Under the low Mach number flow assumption, the axial velocity perturbation is convected instantaneously along the axial direction. In reality, an acoustic perturbation propagating at the sound speed $c=340 \mathrm{~m} \cdot \mathrm{s}^{-1}$ leads to a phase shift $\Delta \varphi=2 \pi f d / c \simeq 97.10^{-3} \mathrm{rad}$, which is negligible at $f=350 \mathrm{~Hz}$. The azimuthal velocity perturbation is convected at the bulk flow velocity $u_{b}=14 \mathrm{~m} . \mathrm{s}^{-1}$ in the annular injection channel. This leads to a phase shift $\Delta \varphi$ that linearly increases with the distance from the exit of the swirler [45]:

$$
\Delta \varphi \simeq 2 \pi \frac{f d}{u_{b}}
$$

For the present case, Eq. 1 yields a phase shift $\Delta \varphi$ of 2.32 rad at $f=350 \mathrm{~Hz}$, a value which is only $5 \%$ larger than the value found in the cold flow simulation. For all the reactive

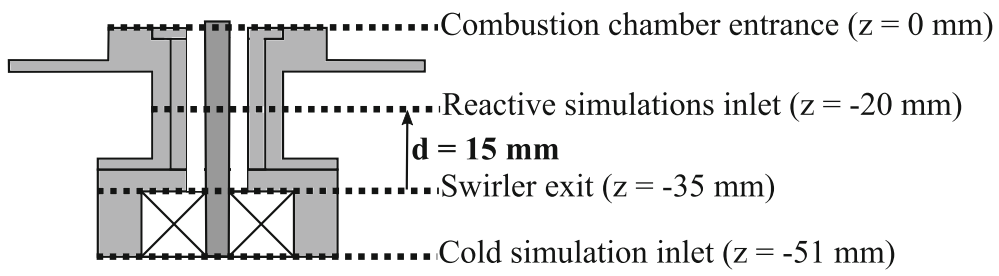

Fig. 3 Geometry of the injection unit and locations of the planes of interest 
Fig. 4 Normalized phase averaged velocity signal at $z=-35 \mathrm{~mm}$ (solid blue line) and $z=-20 \mathrm{~mm}$ (dashed red lines) for a forcing frequency of $f=350 \mathrm{~Hz}$ and $u_{z}^{\prime} / \bar{u}_{z}=0.17$. Top: axial velocity $u_{z} / \bar{u}_{z}$. Bottom: azimuthal velocity $u_{\theta} / \bar{u}_{\theta}$

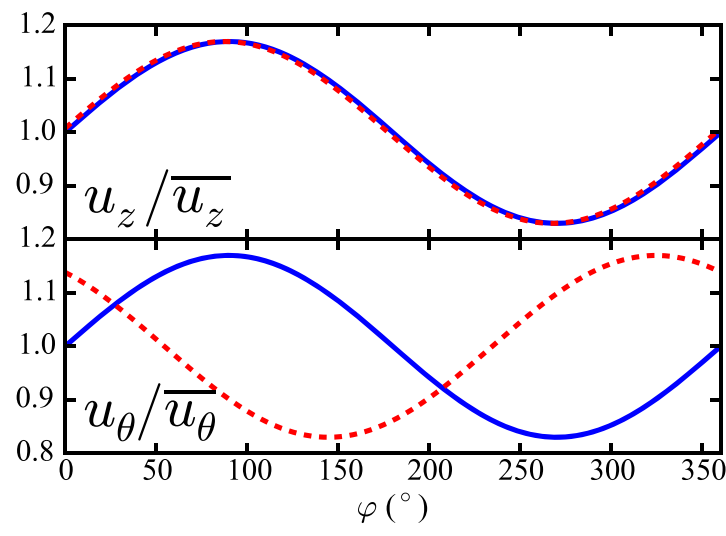

flow simulations presented in this work, Eq. 1 is used to set the phase shift between the azimuthal and axial velocity perturbations at the numerical domain inlet of CD1.

\section{Flame Describing Function}

The FDF is a nonlinear transfer function linking heat release rate fluctuations $\dot{Q}^{\prime}$ to velocity disturbances $u^{\prime}$ at some location before the flame zone. This frequency response is both a function of the forcing frequency and the disturbance level [6]:

$$
F\left(\omega,\left|u^{\prime}\right|\right)=\frac{\dot{Q}^{\prime} / \overline{\dot{Q}}}{u^{\prime} / \bar{u}}=G\left(\omega,\left|u^{\prime}\right|\right) e^{i \varphi\left(\omega,\left|u^{\prime}\right|\right)}
$$

where $G$ is the gain of the FDF and $\varphi$ its phase. This formulation makes an hypothesis of weak nonlinearity of the flame response to the harmonic velocity disturbance $u^{\prime}$ and only considers the first harmonic $\dot{Q}^{\prime}$ of the flame response. The higher harmonics are not considered. The quantities $\bar{u}$ and $\overline{\dot{Q}}$ denote the time averaged values. In the experiments, the velocity disturbances $u^{\prime}$ are measured with a hot wire located upstream of the inlet of the swirler at $z=-65 \mathrm{~mm}$ (see Fig. 1) in a region where the flow is axial, laminar with a top hat velocity profile. The mean $\overline{\dot{Q}}$ and fluctuating $\dot{Q}^{\prime}$ heat release rate components are deduced from $\mathrm{OH}^{*}$ light intensity $I$ measurements collected over the whole flame volume [3] : $I^{\prime} / \bar{I}=\dot{Q}^{\prime} / \bar{Q}$. In LES, the local filtered heat release rate $\overline{\dot{\omega}_{T}}$ is computed as:

$$
\overline{\dot{\omega}_{T}}=\gamma(\Delta h) \Xi \overline{\dot{\omega}_{T}^{*}}(\tilde{c})
$$

where $\gamma(\Delta h)$ is a parameter introduced to take into account heat losses [41], $\Xi$ is the subgrid scale wrinkling modeled as in [44] and $\overline{\dot{\omega}_{T}^{*}}(\tilde{c})$ is the heat release rate tabulated in the filtered F-TACLES look-up table. The progress variable $\widetilde{c}$ is the solution of a filtered balance equation, as discussed in [40]. The filtered heat release rate $\overline{\dot{\omega}}_{T}$ is integrated over the whole computational domain to obtain the heat release rate $\dot{Q}$ :

$$
\dot{Q}=\int_{V} \overline{\dot{\omega}_{T}} d V
$$


The time averaged value is noted $\overline{\dot{Q}}$ and $\dot{Q}^{\prime}$ corresponds to the Fourier component at the harmonic forcing frequency $f$. The velocity disturbance $u^{\prime}$ is computed in the simulation from the fluctuating mass flow rate. In both experiments and simulations, the FDF is calculated as the ratio between the cross spectral density $P_{y, x}$ of $\dot{Q}$ and $u$ and the power spectral density $P_{x, x}$ of $u$ taken at the forcing frequency, which is then normalized by $\bar{u} / \bar{Q}$ :

$$
F\left(\omega,\left|u^{\prime}\right|\right)=\frac{P_{\dot{Q}, u}}{P_{u, u}} \frac{\bar{u}}{\overline{\dot{Q}}}
$$

The gain $G$ and the phase $\varphi$ of each point of the FDF are respectively determined as the modulus and phase lag of $F$ with respect to the velocity signal at the hot wire location:

$$
\begin{aligned}
G\left(\omega,\left|u^{\prime}\right|\right) & =\left|F\left(\omega,\left|u^{\prime}\right|\right)\right| \\
\varphi\left(\omega,\left|u^{\prime}\right|\right) & =\arg \left(F\left(\omega,\left|u^{\prime}\right|\right)\right)
\end{aligned}
$$

Experiments are conducted for harmonic flowrate excitations from $f=10 \mathrm{~Hz}$ to $370 \mathrm{~Hz}$ with $10 \mathrm{~Hz}$ steps and two forcing levels $u^{\prime} / \bar{u}=0.09$ and $u^{\prime} / \bar{u}=0.17$. Numerically, twelve simulations are performed on CD1 with a mesh including 49 million elements to investigate the flame response at six forcing frequencies for both levels of velocity disturbance. All cases are synthesized in Table 1.

Simulations are carried out on an IBM Blue Gene/Q cluster on 1024 processors. Simulating one period of modulation for the case FT-350-09 takes 2800 CPUh, while for case FT-100-17 it reaches 10000 CPUh.

Table 1 Investigated cases in simulations

\begin{tabular}{llll}
\hline Simulation & $\begin{array}{l}\text { Frequency } \\
(\mathrm{Hz})\end{array}$ & $u^{\prime} / \bar{u}$ & $\begin{array}{l}\text { Number of } \\
\text { elements }\left(\times 10^{6}\right)\end{array}$ \\
\hline FT-100-09 & 100 & 0.09 & 49 \\
FT-100-17 & 100 & 0.17 & 49 \\
FT-160-09 & 160 & 0.09 & 49 \\
FT-160-17 & 160 & 0.17 & 49 \\
FT-160-09-F & 160 & 0.09 & 395 \\
FT-200-09 & 200 & 0.09 & 49 \\
FT-200-17 & 200 & 0.17 & 49 \\
FT-250-09 & 250 & 0.09 & 49 \\
FT-250-17 & 250 & 0.17 & 49 \\
FT-300-09 & 300 & 0.09 & 49 \\
FT-300-17 & 300 & 0.17 & 49 \\
FT-350-09 & 350 & 0.09 & 49 \\
FT-350-17 & 350 & 0.17 & 49 \\
FT-350-09-F & 350 & 0.09 & 395 \\
& & & 49
\end{tabular}




\section{Results}

\subsection{Flame Describing Function}

Results of the simulations described in Table 1 are compared to experimental data for the FDF gain and phase lag in Fig. 5. The simulated phase lag is in fairly good agreement with experiments for the range of frequencies investigated. Results better match for the largest perturbation level $u^{\prime} / \bar{u}=0.17$. The inflection point of the phase lag curve in the experiments at $f=160 \mathrm{~Hz}$ is however not reproduced by the simulations at $u^{\prime} / \bar{u}=0.09$.

The quality of the FDF gain prediction by the LES is much more sensitive to the excitation frequency. Slight differences are observed at $f=100,200,250,300$ and $350 \mathrm{~Hz}$, with a significant over-prediction of the FDF gain for an excitation at $f=160 \mathrm{~Hz}$. Figure 5 shows that the measured FDF gain curves feature a low response at $f=160 \mathrm{~Hz}$ and a high value at $f=350 \mathrm{~Hz}$. Differences between simulations and measurements are interpreted in the following by analyzing the flow and flame responses at these two forcing frequencies.

\subsection{Flow and flame dynamics}

Figures 6 and 7 (left side of each panel) show the experimental data gathered for five phase angles regularly distributed in a modulation period at $f=350 \mathrm{~Hz}$ and $f=160 \mathrm{~Hz}$, respectively. These images show on the left, phase conditional averages of the velocity field superimposed on the probability of presence of hot burnt gases in the axial plane of the burner. Two types of arrows are used to emphasize differences between the large velocities reached by the flow in the swirling jet (blue arrows) and the smaller velocities in the outer recirculation zones of the flow (green arrows). The trace of the $\mathrm{OH}^{*}$ chemiluminescence signal in the same axial plane (after Abel deconvolution) is plotted on the right together with iso-levels of probability of presence of burnt gases. The convection velocities of large

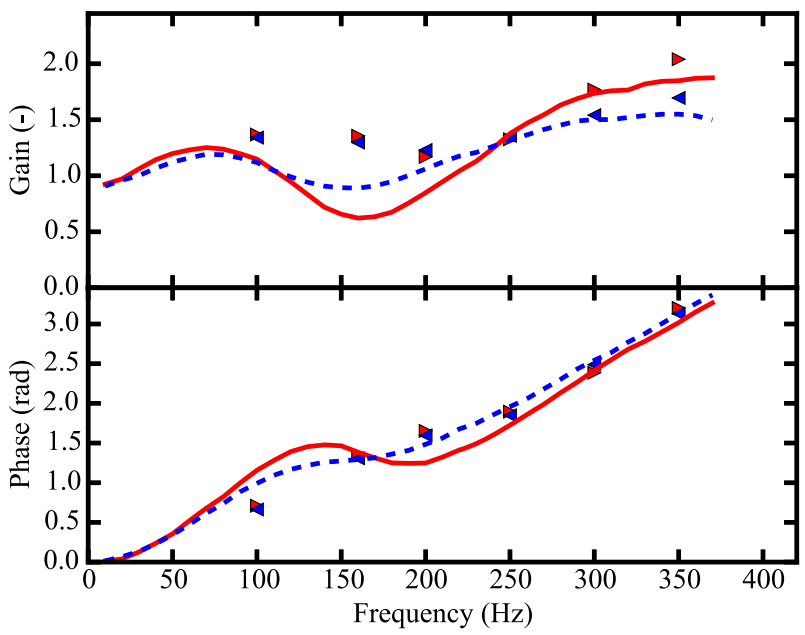

Fig. 5 FDF gain (top) and phase lag (bottom). Experiments : $u^{\prime} / \bar{u}=0.09$ (red solid line), $u^{\prime} / \bar{u}=0.17$ (blue dashed lines). Simulations obtained with the 49 million element mesh: $u^{\prime} / \bar{u}=0.09$ (red items), Red items: $u^{\prime} / \bar{u}=0.17$ (blue items) 

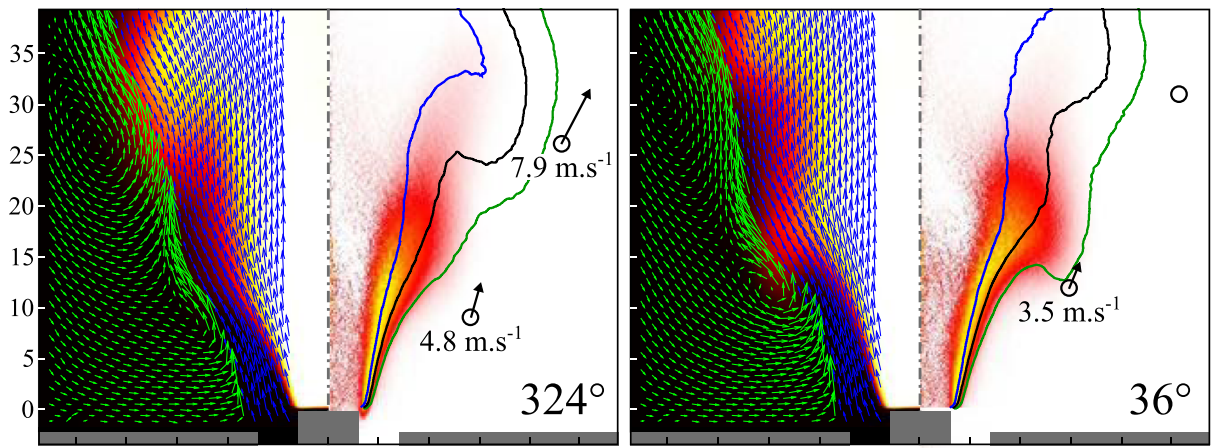
$\begin{array}{ccc}0 & 0.2 \quad 0.4 \quad 0.6 \quad 0.8 \\ \text { Hot burnt gases } \\ \text { probability }\end{array}$

$\begin{array}{cc}\longrightarrow 5 \mathrm{~m} \cdot \mathrm{s}^{-1} & -0.9 \\ \longrightarrow 30 \mathrm{~m} \cdot \mathrm{s}^{-1} & -0.5 \\ \text { Velocity } & -0.1 \\ & \begin{array}{c}\text { Hot burnt gases } \\ \text { probability }\end{array}\end{array}$

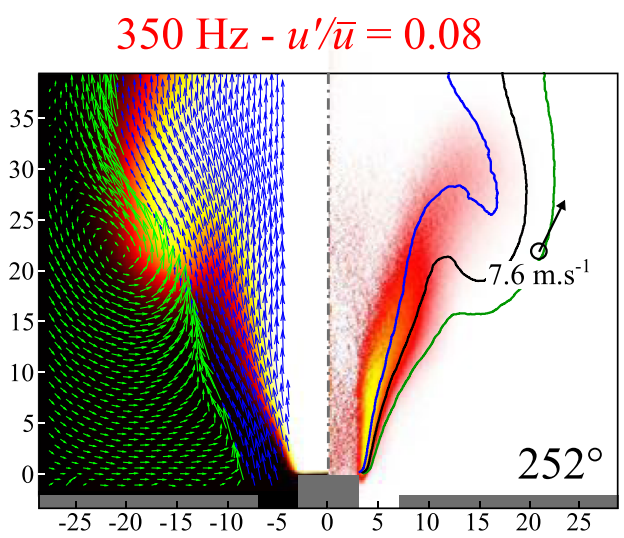

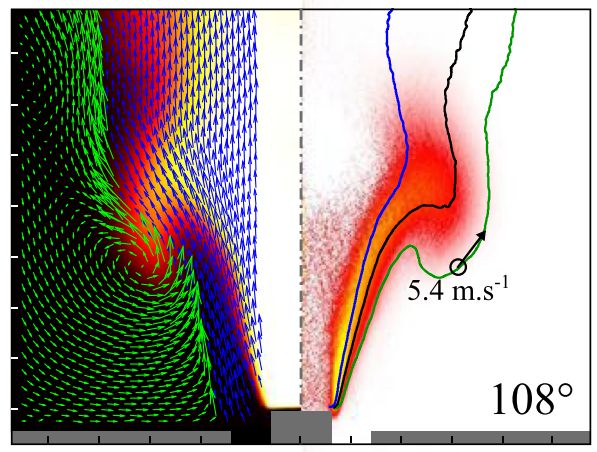

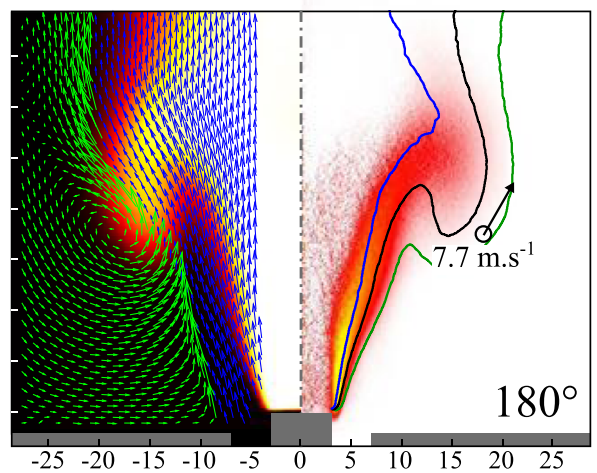

Fig. 6 Modulation at $f=350 \mathrm{~Hz}$ and $u^{\prime} / \bar{u}=0.08$. Experimental results for the phase-averaged velocity field, probability of presence of hot burnt gases (left) and $\mathrm{OH}^{*}$ intensity with iso-contours of hot burnt gas probability (right) at five regularly distributed phase angles in the oscillation cycle. The position of the center of a large vortical structure and its convection velocity are indicated as a circle and an arrow. Dimensions are indicated in millimeters

coherent structures detected by post-processing and the velocity field are also indicated together with the position of the center of these vortices.

For the case at $f=350 \mathrm{~Hz}$ shown in Fig. 6, $\varphi=324^{\circ}$ corresponds to the first phase, out of the five examined, where a large coherent vortical structure is detected in the outer shear (mixing) layer of the swirling jet with a center at about $9 \mathrm{~mm}$ above the top of the central insert and which is convected at a velocity $4.8 \mathrm{~m} . \mathrm{s}^{-1}$. Note that two consecutive vortices corresponding to two consecutive modulation periods are visible in the field of view for this phase. The second one with a center at about $25 \mathrm{~mm}$ moves faster at a speed of $7.9 \mathrm{~m} . \mathrm{s}^{-1}$. 

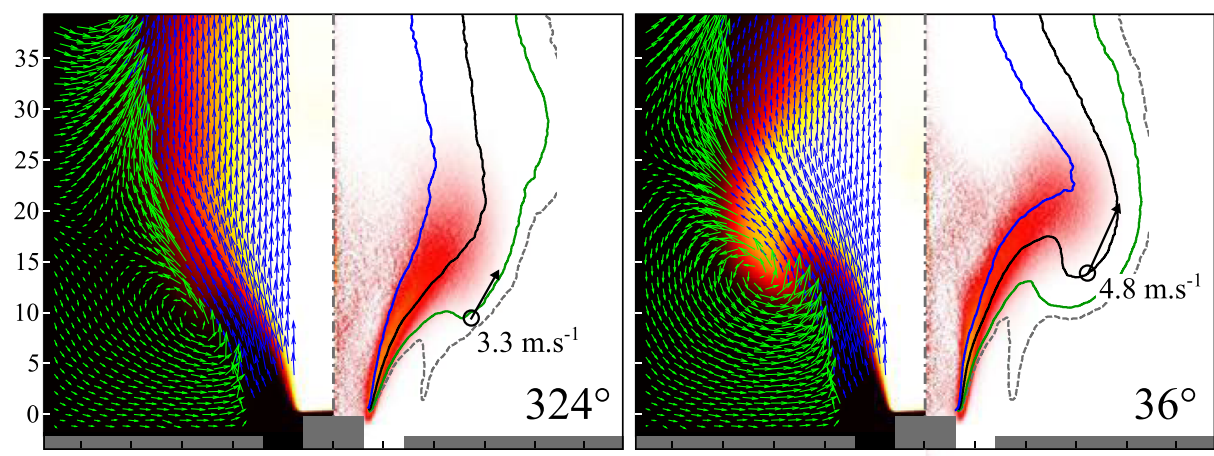
$\begin{array}{llllll}0 & 0.2 & 0.4 & 0.6 & 0.8\end{array}$ Hot burnt gases probability

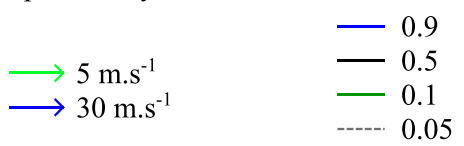

Velocity $\begin{array}{lllllll}1 & 0 & 0.2 & 0.4 & 0.6 & 0.8 & 1\end{array}$

$\mathrm{OH}^{*}$ intensity (a.u.)

Hot burnt gases probability

$$
160 \mathrm{~Hz}-u^{\prime} / \bar{u}=0.12
$$
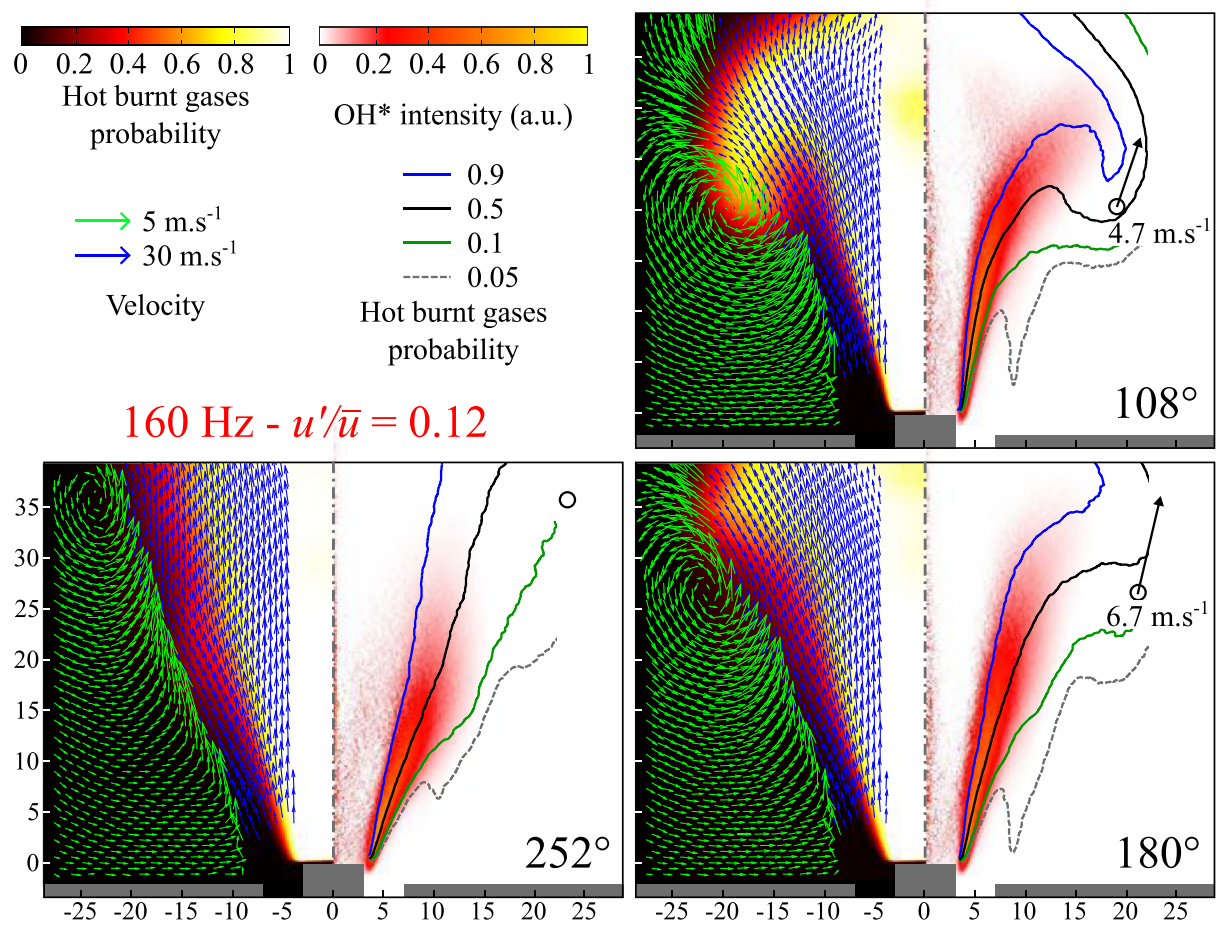

Fig. 7 Modulation at $f=160 \mathrm{~Hz}$ and $u^{\prime} / \bar{u}=0.12$. Experimental results for the phase-averaged velocity field, probability of presence of hot burnt gases (left) and $\mathrm{OH}^{*}$ intensity with isocontours of hot burnt gas probability (right) at five regularly distributed phase angles in the oscillation cycle. The position of the center of a large vortical structure and its convection velocity are indicated as a circle and an arrow. Dimensions are indic ated in millimeters

The loop sequence $324^{\circ} \leq \varphi \leq 324^{\circ}+360^{\circ}$ allows following the growth and downstream convection of each coherent structure in the mixing layer along the flame brush until it reaches the flame tip at a phase lag around $\varphi=252^{\circ}$. The right sides in Fig. 6 show the measured $\mathrm{OH}^{*}$ emission intensity (red/orange colormap) after an inverse Abel transform is applied on the phase averaged images. These images ease analyzing interactions between the flame and the large vortical structures during a modulation period.

At a forcing frequency $f=350 \mathrm{~Hz}$ and a forcing level $u^{\prime} / \bar{u}=0.08$, the flame brush in Fig. 6 progressively rolls up around the large vortical structure at $\varphi=36^{\circ}$ and $108^{\circ}$ 
and the flame length increases, before the flame is disrupted between phases $\varphi=108^{\circ}$ and $180^{\circ}$ when the vortical structure reaches the flame tip. The flame then rapidly shrinks at phases $\varphi=180^{\circ}$ and $252^{\circ}$. This mechanism is similar to the one observed for laminar V flames submitted to harmonic flow modulations [46]. This process leads to large flame surface area fluctuations during a modulation period and is responsible for the large FDF gain observed at this forcing frequency in Fig. 5. The probability to find hot burnt gases ( $T \geq 1800 \mathrm{~K}$ [29]) deduced from OH-LIF measurements is also shown in Fig. 6. The circular pocket of hot burnt gases entrained around each vortical structure during a modulation period confirms the flame/vortex roll-up process taking place in the external shear layer of the swirling flow. One may also note an interesting feature. The convection velocity of these structures depends on the height above the burner and features a complex behavior [47]. With the data available, the vortical structure seems to first decelerate before reaccelerating. This observation is in contrast with simplified assumptions made in many low order models [48, 49].

Figure 7 shows the experimental results found at a lower forcing frequency $f=160 \mathrm{~Hz}$, but a slightly higher forcing level $u^{\prime} / \bar{u}=0.12$. The sequence of phase conditioned data again allows following the growth and downstream convection of a large vortical structure shed from the injector lip during a modulation period. In comparison to results at $f=$ $350 \mathrm{~Hz}$ at $u^{\prime} / u=0.08$, the vortex consistently features a larger diameter due to the lower forcing frequency and the higher modulation level [47]. A large circular pocket of hot burnt gases entrained by the vortical structure is again visible for this forcing frequency in each image. However, the $\mathrm{OH}^{*}$ emission data also reveal that the flame tip is less bent by these large structures during a modulation period at $f=160 \mathrm{~Hz}$ than at $f=350 \mathrm{~Hz}$. Therefore, it can be concluded that the flame/vortex roll-up process is less efficient for $f=160 \mathrm{~Hz}$ and $u^{\prime} / \bar{u}=0.12$ than for an excitation at $f=350 \mathrm{~Hz} u^{\prime} / \bar{u}=0.08$. This observation highlights the determinant role of the complex flame/vortex roll-up process taking place in the flame response. A larger and more energetic structure does not necessarily leads to a stronger flame response.

One may already suggest that the vortical structure produced for the forcing conditions explored in Fig. 7 is too large in comparison to the flame length to maximize the flame/vortex roll-up process, even though this structure is energetic enough to recirculate the hot burnt gases. The role of these coherent structures in the peak value of the FTF gain has already been highlighted for the response of laminar $\mathrm{V}$ flames [46] and for the response of swirling flames $[9,47]$. Oberleithner et al. [47] showed that the receptivity of the shear layer is an essential feature to understand the streamwise growth of these coherent structures. They found that the FDF gain of their swirling flame is correlated to the receptivity of the shear layer when the forcing frequency and amplitude are varied. It is found here that the flame/vortex interaction needs also to be considered and the process is not purely hydrodynamic. Interaction of a large coherent structure with the flame may also lead to a low heat release rate response.

A close examination of Figs. 6 and 7 also reveals that the flame angle fluctuates at the base of the central rod during a modulation period. These fluctuations are difficult to see in these images and are better revealed in Fig. 15, which is later discussed. They are larger at $f=160 \mathrm{~Hz}$ than at $f=350 \mathrm{~Hz}$ and are attributed to temporal fluctuations of the swirl number $[3,50]$. For an excitation at $f=160 \mathrm{~Hz}$, the flame motion is controlled by two distinct mechanisms : flame/vortex roll-up and swirl number fluctuations. It has been shown in [3] that interferences between these two mechanisms are responsible for a drop of the FDF gain. It is suggested here that the efficiency of the flame/vortex interaction needs also to be considered to understand the observed drop of the flame response at $f=160 \mathrm{~Hz}$. 


\subsection{Comparison with simulations}

The FDF only yields an integrated information on the global flame response to the flow perturbations. A deeper analysis is made in this section by comparing the measured and computed velocity fields, the $\mathrm{OH}^{*}$ emission and heat release distributions and the hot burnt gas distributions in the experiments and simulations when the flow is modulated. The $\mathrm{OH}^{*}$ intensity distribution is here again directly compared to numerical fields of volumetric heat release rate, as was done for instance in [51]. This hypothesis could be avoided by using a mechanism which includes $\mathrm{OH}^{*}$ species for chemistry tabulation [52]. However as the burner operates in the fully premixed mode and all signals are normalized by their maximum values, the errors induced by this approximation are expected to be limited and will not alter the following analysis. Also, a kinetic scheme with $\mathrm{OH}^{*}$ would need to be validated with the 1D flame solver used here and a new look-up table would be generated with this new species.

The left columns in Figs. 8 and 9 show images with the measured phase averaged $\mathrm{OH}^{*}$ emission distribution plotted on the left and the phase averaged heat release rate calculated in the simulation on the right for excitations at $f=350 \mathrm{~Hz}$ and $f=160 \mathrm{~Hz}$, respectively. The right columns in these figures compare the measured (on the left side) and computed (on the right side) phase averaged hot burnt gas probability of presence superimposed to the corresponding velocity fields. These comparisons are conducted for five phases in the modulation cycle. One recalls that the phase lag $\varphi$ in these figures is indicated with respect to the velocity signal measured at the hot wire location.

Regardless of the forcing frequency and the phase in the forcing cycle, the experimental and numerical data are close in Figs. 8 and 9. The position and size of the large vortical structures convected in the outer shear layer of the swirling jet are well reproduced at each phase in the cycle. The shape and size of the recirculating hot burnt gases pockets entrained by these vortical structures are also well captured by the LES. Overall, the shape of the flame brush at the different phases in the forcing cycle is also fairly well predicted by the simulations in Figs. 8 and 9. For $f=350 \mathrm{~Hz}$, the large flame/vortex roll-up process is reproduced by the simulations for the phases $36^{\circ} \leq \varphi \leq 180^{\circ}$ where the vortex interacts with the flame tip. For $f=160 \mathrm{~Hz}$, the calculated flame/vortex roll-up process also appears less intense in Fig. 9, which is consistent with the experimental observations.

The velocity fields calculated in the simulations through the axial plane of the burner and the PIV data are extracted at heights $z=5,15,25$ and $35 \mathrm{~mm}$ above the combustion chamber dump plane and are plotted in Figs. 10 and 11. The experimental values are masked for $x<5 \mathrm{~mm}$ because flow seeding is not high enough to provide statistically meaningful information in the central region of the flow. The numerical values are kept in this region to highlight the evolution of the flow in the inner recirculation zone. These additional figures confirm that the shape of the velocity profiles for both components are the same between experiments and simulations at all heights $z$ and phases $\varphi$. There are however differences in the peak values reached by the perturbed flow in experiments and simulations. The largest differences are observed at $x \sim 10 \mathrm{~mm}$ in the outer shear layer of the swirling flow.

One may identify other differences between experiments and simulations in Figs. 8 and 9. The first one is the presence of residual heat release in the outer shear layer of the swirling jet in the simulations. This difference in volumetric heat release distribution can also be observed for the unperturbed flame shown in Fig. 2. The second one is the difference between the axial expansion of the zones with large heat release rate values. Overall, the flame appears taller in the simulations compared to experimental observations. The largest differences are observed for the case at $f=350 \mathrm{~Hz}$ and $\varphi=252^{\circ}$ and $\varphi=324^{\circ}$ in Fig. 8 . 

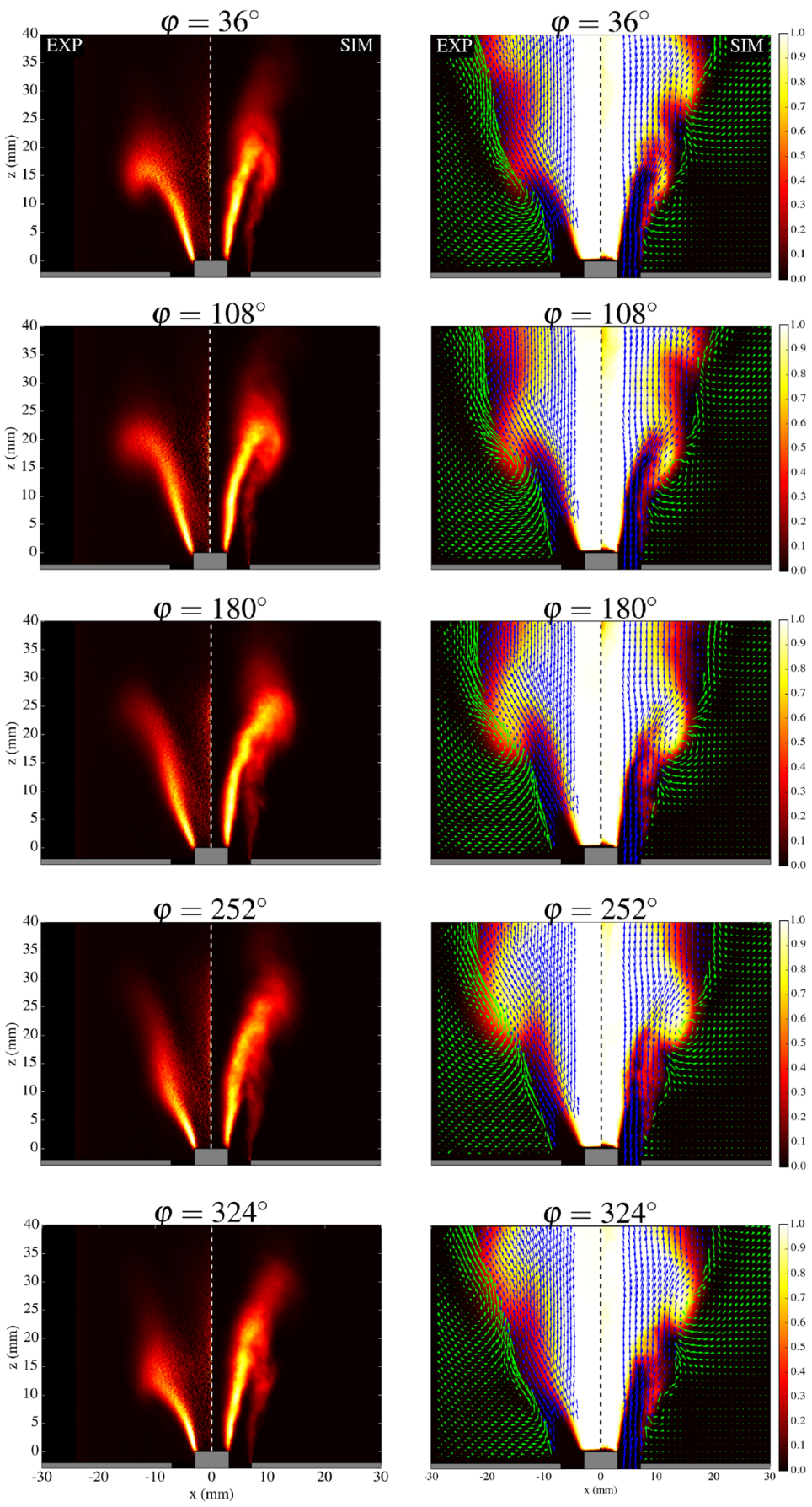

Fig. 8 First column: phase-averaged measured $\mathrm{OH}^{*}$ emission (left) and computed heat release rate (right). Values are normalized by the maximum. Second column: measured (left) and computed (right) phaseaveraged velocity field and hot burnt gas probability of presence. Results are presented for five phase angles of the modulation period. Case FT-350-09 at $f=350 \mathrm{~Hz}$ and $u^{\prime} / \bar{u}=0.09$ in Table 1 

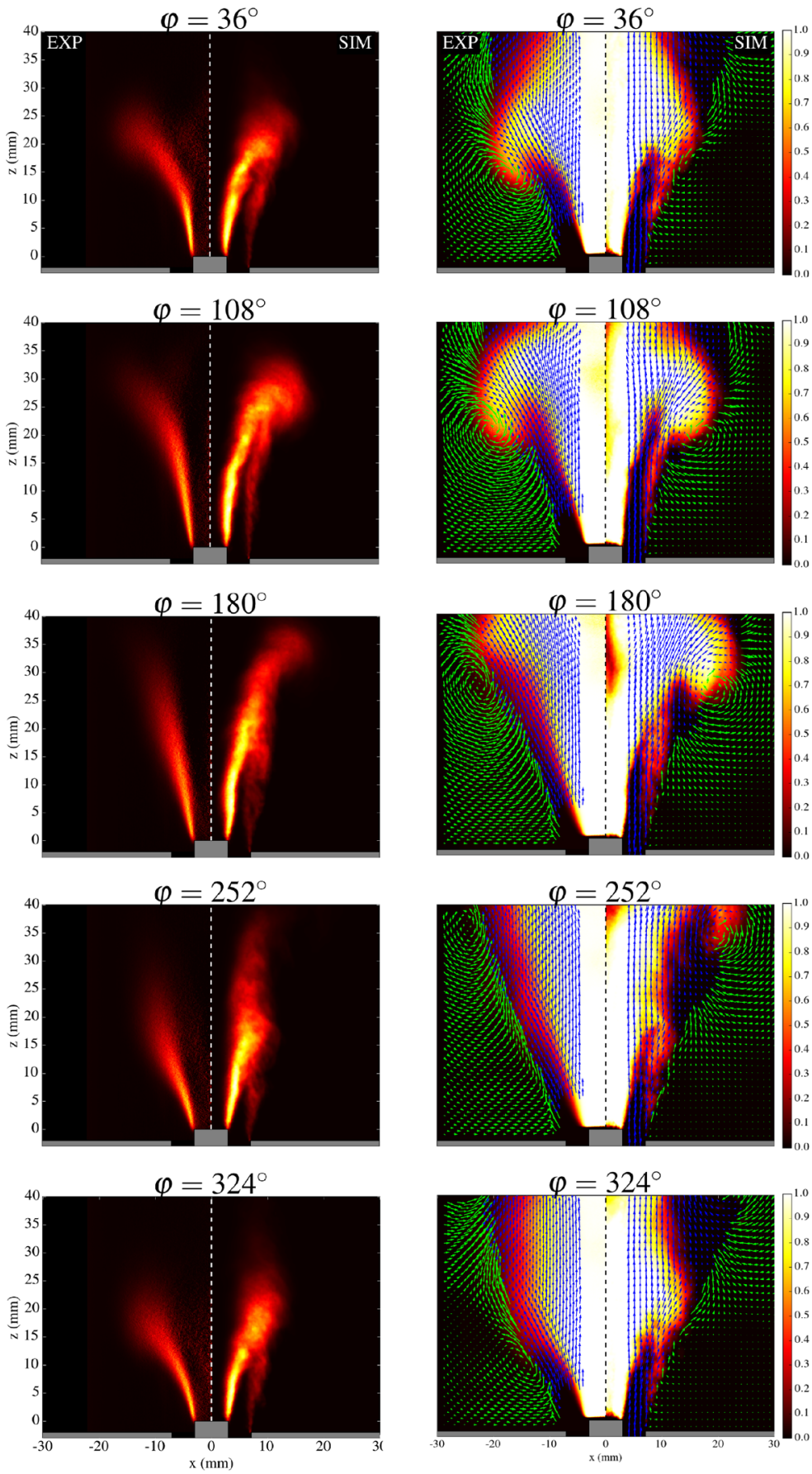

Fig. 9 First column: phase-averaged measured $\mathrm{OH}^{*}$ emission (left) and computed heat release rate (right). Values are normalized by the maximum. Second column: measured (left) and computed (right) phaseaveraged velocity field and hot burnt gas probability of presence. Results are presented for five phase angles of the modulation period. Case FT-160-17 at $f=160 \mathrm{~Hz}$ and $u^{\prime} / \bar{u}=0.17$ in Table 1 

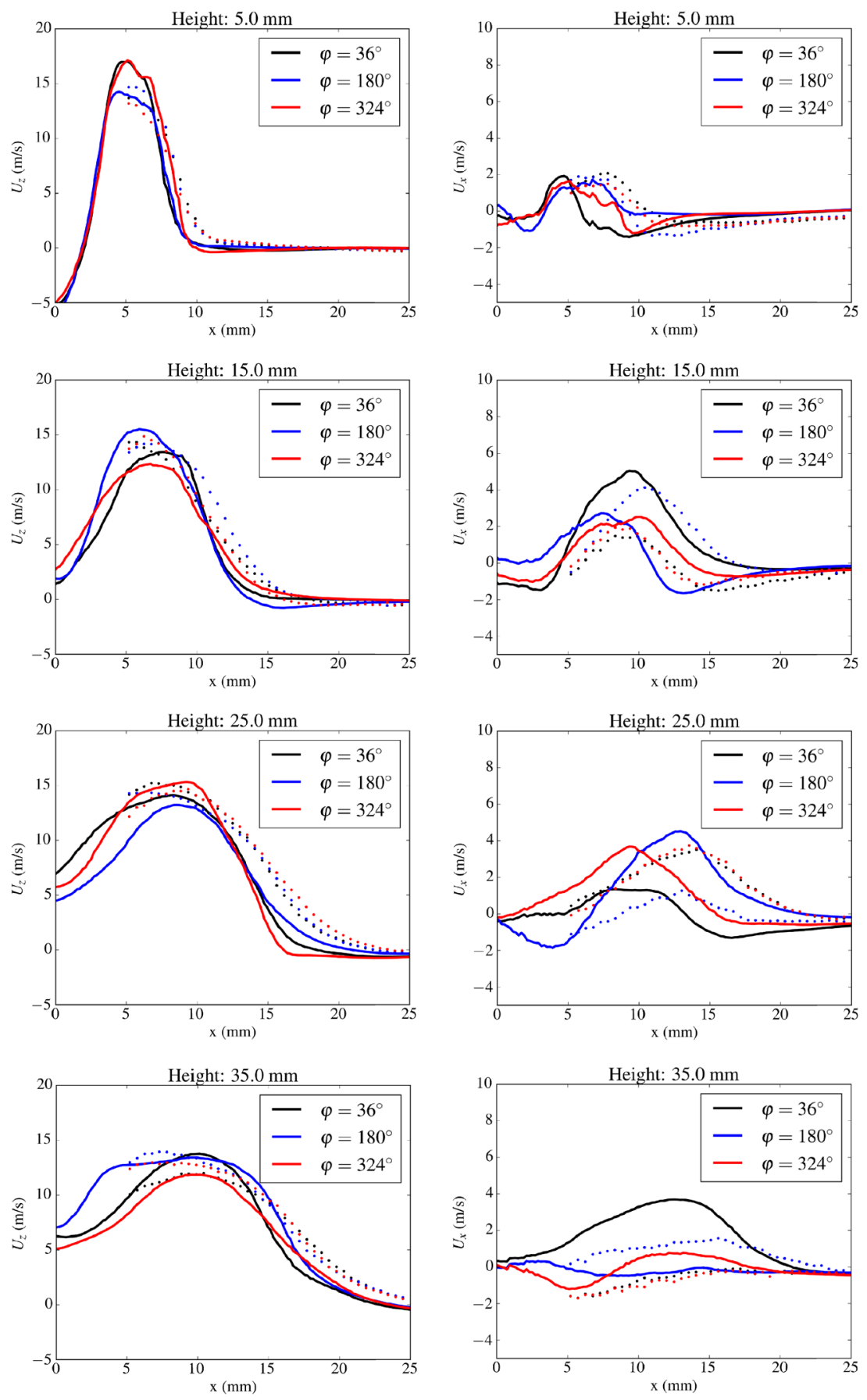

Fig. 10 First column: measured (symbols) and computed (solid lines) phase-averaged axial velocity $u_{z}$. Second column: measured (symbols) and computed (solid lines) phase-averaged radial velocity $u_{x}$. Results are presented for three phase angles of the modulation period and different heights $z$. Case FT-350-09 at $f=350 \mathrm{~Hz}$ and $u^{\prime} / \bar{u}=0.09$ in Table 1 

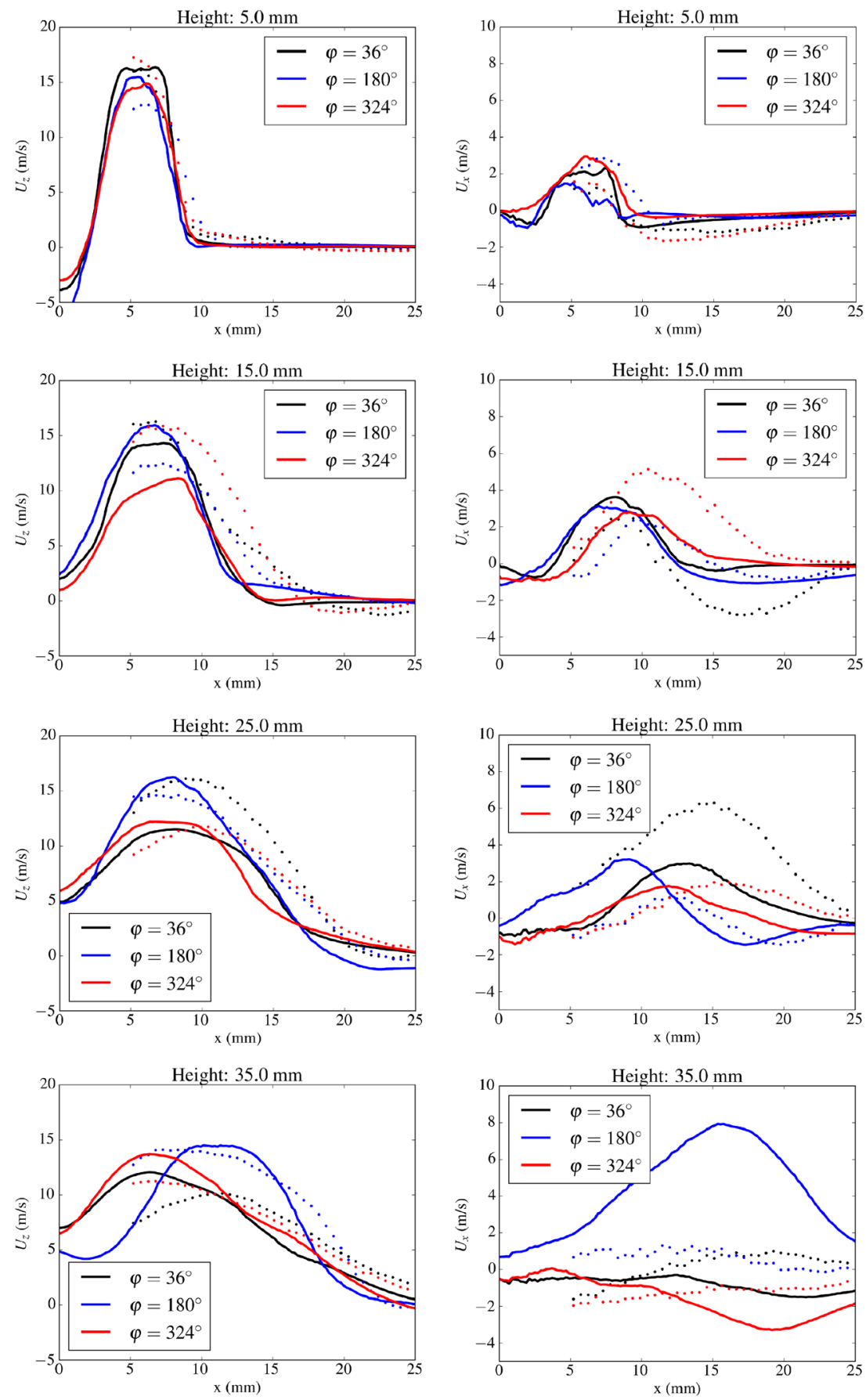

Fig. 11 First column: measured (symbols) and computed (solid lines) phase-averaged axial velocity $u_{z}$. Second column: measured (symbols) and computed (solid lines) phase-averaged radial velocity $u_{x}$. Results are presented for three phase angles of the modulation period and different heights $z$. Case FT-160-17 at $f=160 \mathrm{~Hz}$ and $u^{\prime} / \bar{u}=0.17$ in Table 1 
It is known that the flame angle and flame height are important features that affect the flame frequency response to flow perturbations [48]. Therefore, it is worth examining if differences between the measured and computed FDF gain can be attributed to differences of the observed and simulated flame shapes.

To this end, data are further analyzed by splitting the images in eight interrogation regions of $5 \mathrm{~mm}$ each, as shown in Fig. 12. Layer Z05 denotes the region between the rod tip and $5 \mathrm{~mm}$ above. Layer Z10 comprises the region between $5 \mathrm{~mm} \leq z \leq 10 \mathrm{~mm}$ and so on. Figures $13(f=350 \mathrm{~Hz})$ and $14(f=160 \mathrm{~Hz})$ compare, for each of these layers, the measured (left) and computed (right) evolutions of the phase averaged integral of $\mathrm{OH}^{*}$ intensity (measurements) and heat release rate (simulations) calculated over the region covered by the layer as a function of the phase in the forcing cycle. Values are normalized by the average value obtained in each layer during a modulation period.

Results in Fig. 13 are analyzed first for an excitation at $f=350 \mathrm{~Hz}$. For layers Z05, Z10, Z15, Z20, Z25 and Z30, which correspond to small and intermediate distances from the injector nozzle, agreement between the measured $\mathrm{OH}^{*}$ intensity $I / \bar{I}$ and heat release rate $\dot{Q} / \bar{Q}$ deduced from the simulation is good. The simulation reproduces well the shape of the measured signal both in phase and amplitude in the different interrogation windows. Agreement however worsens when results are compared for the upper layers Z35 and Z40. Both the amplitude and the phase lag of the measured $I / \bar{I}$ and computed $\dot{Q} / \bar{Q}$ signals differ in these regions. These differences have however a limited impact on the global flame response and its FDF as shown in Fig. 5 at $f=350 \mathrm{~Hz}$ because the relative fluctuations $\dot{Q} / \bar{Q}$ remain relatively weak in the regions Z35 and Z40 in Fig. 13 compared to the ones found at Z25 and Z20 which are better reproduced by the simulations.

Results in Fig. 14 for an excitation at $f=160 \mathrm{~Hz}$ are now analyzed. Except for layer $\mathrm{Z} 20$, the shape of the relative fluctuation $I / \bar{I}$ observed for the $\mathrm{OH}^{*}$ signal in the experiment is well reproduced by the relative heat release rate fluctuation $\dot{Q} / \bar{Q}$ found in the LES. The amplitude of these signals is also well reproduced by the LES for layers Z05, Z10, Z15, Z20 and Z25. However, simulations largely over-predict the amplitude of heat release rate

Fig. 12 Definition of interrogation regions

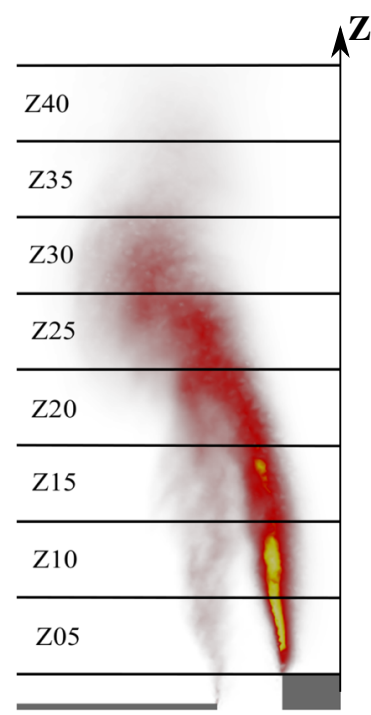



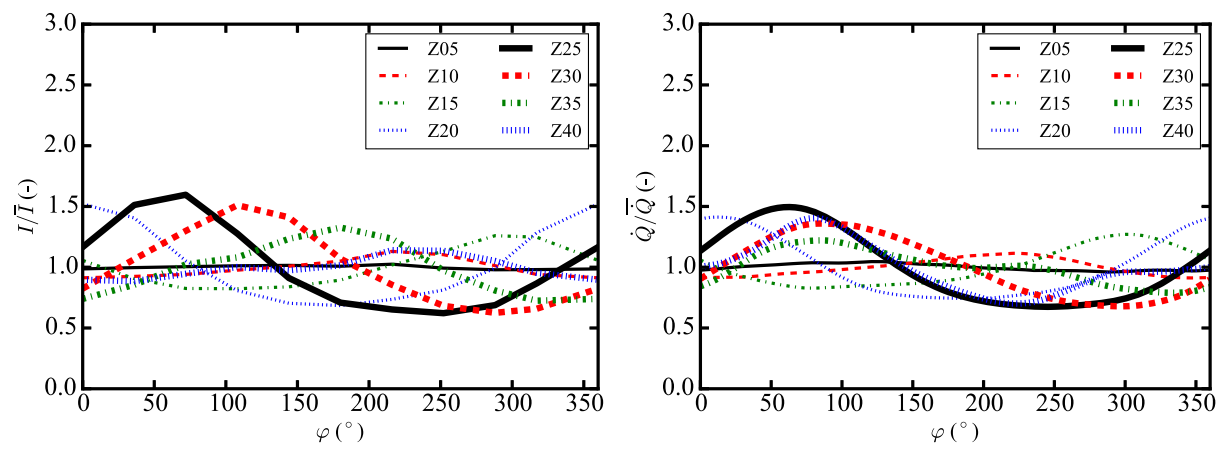

Fig. 13 Evolution of the normalized phase-averaged integral of heat release rate for each layer defined in Fig. 12 during a modulation period. Left: experiments. Right: simulations. Case FT-350-09 at $f=350 \mathrm{~Hz}$ and $u^{\prime} / \bar{u}=0.09$ in Table 1

fluctuations in layers Z30, Z35 and Z40, corresponding to the upper part of the flame. Because the flame stabilizes with a V-shape, the upper parts of the flame comprise a large fraction of the total flame surface area and contribute significantly to the total heat release rate oscillation. Therefore, over-predicting the amplitude of heat release rate fluctuations in these regions is likely to have a significant impact on the predicted FDF. This could explain why the FDF gain is largely over-predicted by the simulation at the forcing frequency $f=$ $160 \mathrm{~Hz}$ in Fig. 5.

The previous figures reveal that heat release rate fluctuations are poorly predicted by the simulation in the upper region of the flame at both forcing frequencies $f=350 \mathrm{~Hz}$ due to phase phase mismatch between the experimental and numerical signals and at $f=160 \mathrm{~Hz}$ due to an amplitude mismatch, but LES predicts the correct FDF gain for an excitation at $f=350 \mathrm{~Hz}$ and over-predicts it for $f=160 \mathrm{~Hz}$ in Fig. 5. To better understand this behavior, Fig. 15 plots the minimum and maximum values of the heat release rate $\dot{Q}$ and $\mathrm{OH}^{*}$ light intensity $I$ signals found in each interrogation region over a forcing cycle. This comparison gives an indication on how well the envelope of the flame motion is reproduced by the simulation as a function of the distance to the injector outlet.
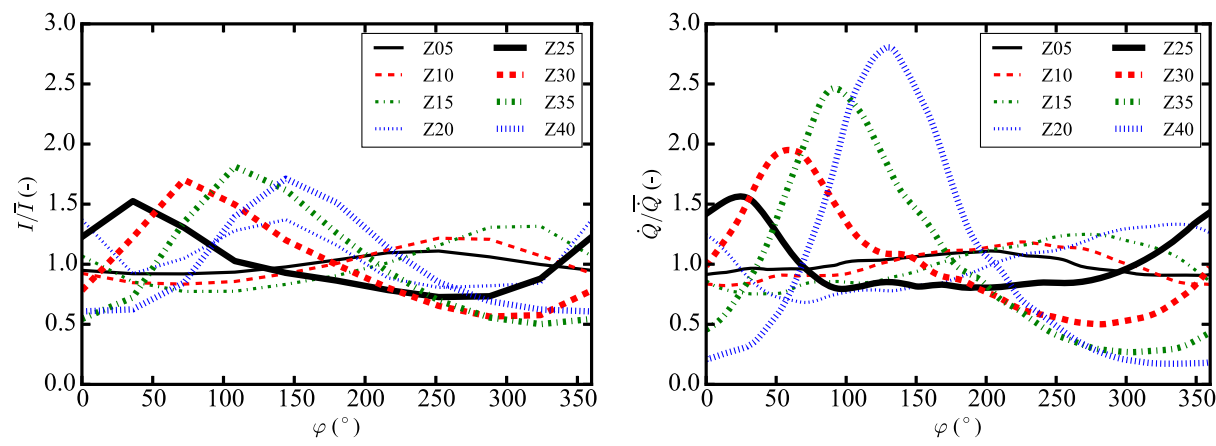

Fig. 14 Evolution of the normalized phase-averaged integral of heat release rate for each layer defined in Fig. 12 during a modulation period. Left: experiments. Right: simulations. Case FT-160-17, $f=160 \mathrm{~Hz}$ and $u^{\prime} / \bar{u}=0.17$ in Table 1 

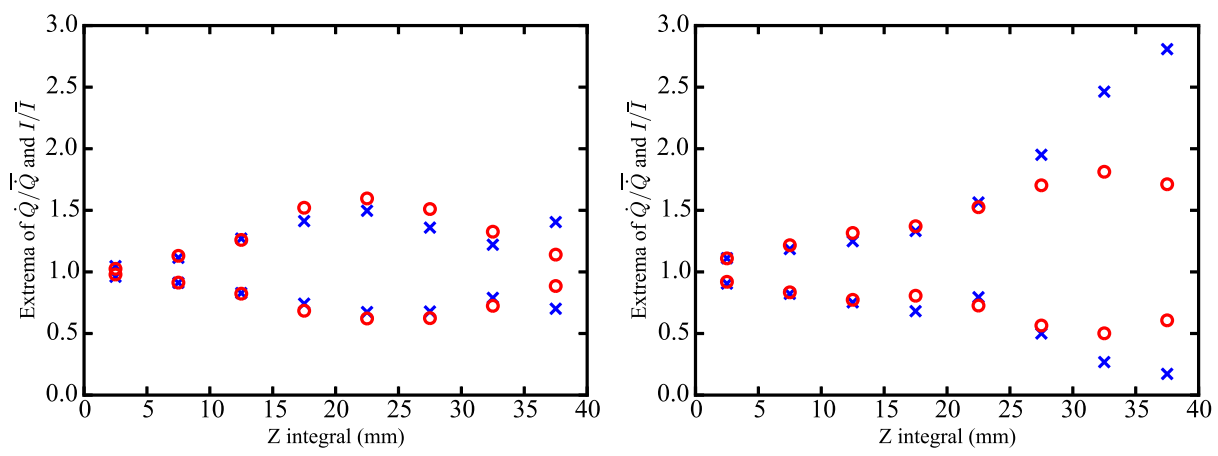

Fig. 15 Extrema of the normalized phase-averaged integral heat release rate in each layer defined in Fig. 12 as a function of the downstream position. Blue crosses: simulations. Red circles: experiments. Left : case FT-350-09. Right: case FT-160-17

Results for $f=350 \mathrm{~Hz}$ and $u^{\prime} / \bar{u}=0.09$ are shown at the left in Fig. 15. One first sees that the envelope of the perturbed flame brush does not move close to the flame anchoring region, meaning that the flame root angle does not vary in this region at this forcing condition. The flame brush envelope then starts to grow further downstream and this motion grows in amplitude with the downstream distance to the rod tip up to the region Z20. At this distance, the amplitude of this motion starts to shrink in the experiments further downstream. These features are well reproduced by the LES, except very close to the flame tip where the LES overestimates the amplitude of the flame brush motion at Z40. As already stated, this has a limited impact on the FDF at $f=350 \mathrm{~Hz}$, because the contribution to the total heat release fluctuation of the signal originating from the flame tip remains relatively weak in this case.

The situation differs in the right graph in Fig. 15 obtained for a forcing frequency $f=$ $160 \mathrm{~Hz}$ and $u^{\prime} / \bar{u}=0.17$. One may already note a sizable motion of the flame brush envelope very close to the flame anchoring region associated to large fluctuations of the flame root angle for this modulation. The envelope of the flame brush motion then also grows with the downstream distance to the rod tip and this is again well reproduced by the simulation up to region Z20. The maximum fluctuation amplitude is reached for Z30 in the experiments. The measured signal then slightly drops at Z40 while the envelope of the simulated signal continuously grows with the distance to the rod tip and reaches the largest oscillations at the flame tip. Therefore, LES does not accurately predict the FDF gain at $f=160 \mathrm{~Hz}$ in Fig. 5 due to a difficulty at reproducing the correct flame tip motion at this forcing condition.

\section{Analysis}

The objective of the following analysis is to identify the reasons which explain the limitation of the LES and to provide routes for improvement. Differences between LES predictions and experiments for the FDF gain and flame motion suggest that turbulent heat release fluctuation prediction should be improved. The data presented in the previous sections also indicate that these differences may be specifically attributed to difficulties in computing the spatial distribution of the heat release rate, particularly near the tip of the flame. This issue potentially derives for errors in determining the correct flame consumption speed, which controls the flame shape and flame height. Different scenarii are now envisaged to explore these issues. 


\subsection{Scenario A: impact of the grid resolution}

Original simulations carried out on a mesh with 49 million elements have been reconducted on a homogeneously refined grid made of 395 million elements for both forcing frequencies $f=160 \mathrm{~Hz}$ and $f=350 \mathrm{~Hz}$. Results for the flame dynamics are not shown here, but one barely sees any difference with the phase averaged fields plotted in Figs. 8 and 9 that were obtained with the original grid. Values for the FDF gain and phase lag obtained on the fine grid are added to the FDF plotted in Fig. 16. The results are a bit improved at $f=350 \mathrm{~Hz}$, but there is no significant difference, meaning that the grid resolution does not improve the quality of the prediction of flame response to flow perturbations and in particular does not help to reduce the difference observed at $f=160 \mathrm{~Hz}$.

\subsection{Scenario B: impact of the subgrid scale flame wrinkling model}

Subgrid scale flame wrinkling directly alters the effective propagation speed of the turbulent flame and may therefore influence the quality of the FDF prediction. In the present formulation, uncertainties in flame wrinkling modeling are mainly concentrated in the estimation of the ad-hoc $\beta$ parameter of the Charlette model [44] given by:

$$
\Xi_{\Delta}=\left(\frac{\Delta}{\delta_{l}^{0}}\right)^{\beta}
$$

where $\Delta$ is the spatial filter width and $\delta_{l}^{0}$ the laminar flame thickness. In the present simulations, the efficiency function $\Xi_{\Delta}$ reaches 2.83. Assuming the saturated form of the Charlette model, changing the $\beta$ value leads to changes of the turbulent flame speed $S_{T}$ as follows:

$$
\frac{S_{T}^{\beta+\Delta \beta}}{S_{T}^{\beta}}=\left(\frac{\Delta}{\delta_{l}^{0}}\right)^{\Delta \beta}
$$

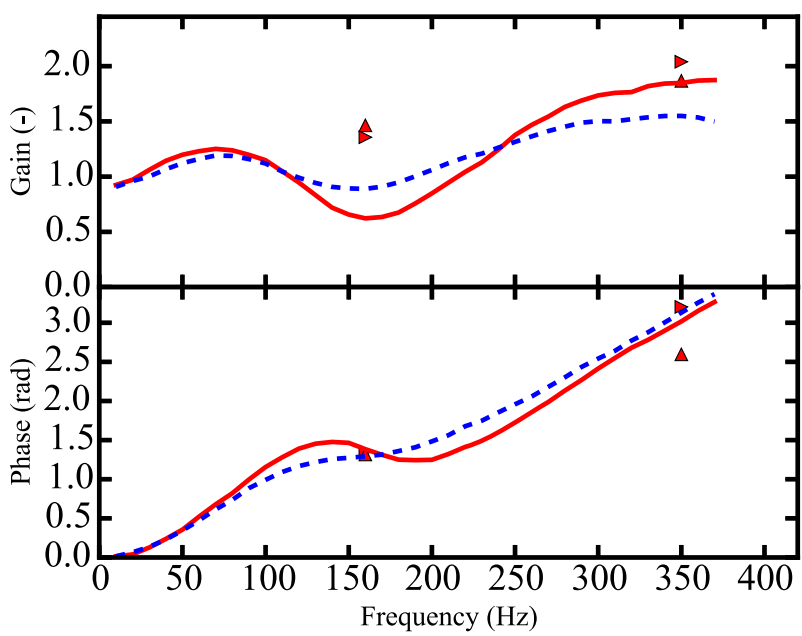

Fig. 16 FDF gain (top) and phase lag (bottom). Experiments : $u^{\prime} / \bar{u}=0.09$ (red solid line), $u^{\prime} / \bar{u}=0.17$ (blue dashed lines). Simulations : $u^{\prime} / \bar{u}=0.09$ (red items). Upward oriented triangles: refined mesh with 395 million elements. Rightward oriented triangles: original mesh with 49 million elements 
Figure 17 compares the flame shapes calculated for $\beta=0.5$ and $\beta=0.6$ with the experimental data in the absence of external forcing. Increasing $\beta$ to 0.6 corresponds to an increase of $20 \%$ of $S_{l, 0}$. According to Eq. 9 , with $\Delta=3.5 \mathrm{~mm}$ and $\delta_{l}^{0}=0.44 \mathrm{~mm}$, this corresponds to an increase of $S_{T}$ approximately equal to $23 \%$. As expected, the flame brush is shorter in Fig. 17 with an increased turbulent flame speed for $\beta=0.6$. The distribution of volumetric heat release rate now also better matches the $\mathrm{OH}^{*}$ distribution in the experiments. The main difference between experiments and these new simulations is now related to the thin residual reaction layer in the outer shear layer of the swirling jet, which is absent in the $\mathrm{OH}^{*}$ experiment, but is still present in the simulated heat release distribution.

This new numerical solution is retained to assess the influence of the $\beta$ parameter on the flame response to a flow modulation at $f=160 \mathrm{~Hz}$ and $u^{\prime} / \bar{u}=0.17$ (case FT-160-17 in Table 1). This case is chosen because the largest differences with experiments are observed for the FDF gain at $f=160 \mathrm{~Hz}$. The same procedure is applied for the flow modulation, the only change being the increase of $\beta$ from 0.5 to 0.6 . As shown in Fig. 18, the calculated FDF gain with $\beta=0.6$ is not improved in any way with respect to the experimental data and to the previous simulations made with $\beta=0.5$.

This test shows that the $\beta$ parameter cannot explain the discrepancy of the FDF gain observed at $f=160 \mathrm{~Hz}$ between experiments and simulations. The flame dynamics is, for this configuration, not very sensitive to the subgrid scale flame modeling. As $\Xi$ decreases with mesh refinement, this conclusion is somehow consistent with scenario A, where it has been shown that results are not very sensitive to the grid size. This result also reveals that despite the flame length is better reproduced by LES for the unforced case, the flame response observed in the experiments is still not recovered. This means that the role of the residual reaction rate observed in the simulations for $\beta=0.5$ or $\beta=0.6$ in the outer shear layer of the swirling flow may have a major effect on the flame response to acoustic modulations and its FDF at $f=160 \mathrm{~Hz}$.
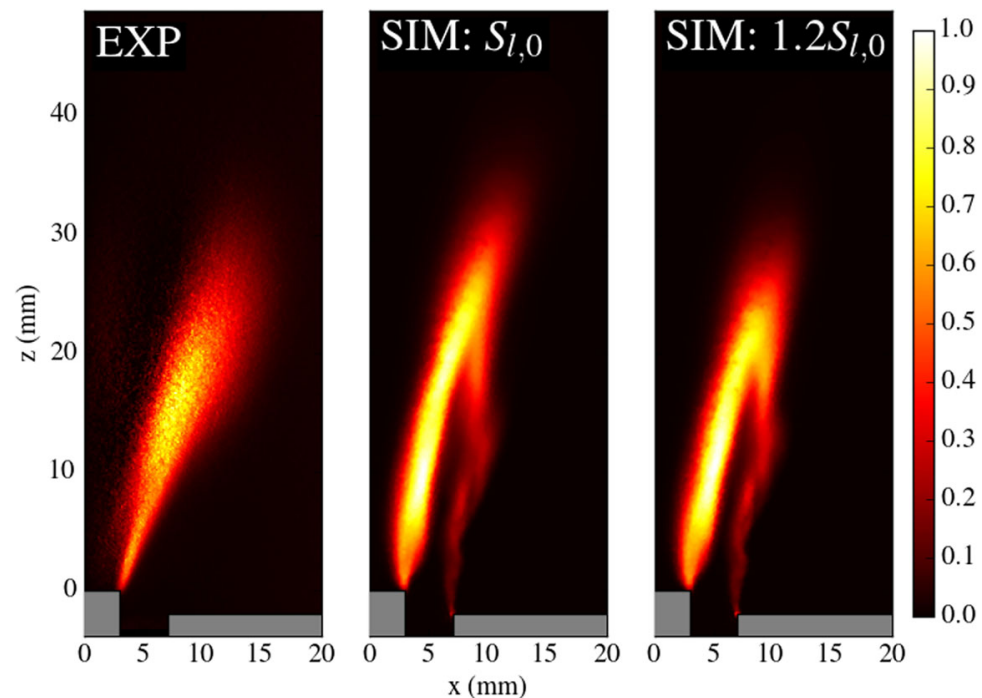

Fig. 17 Comparison of the measured $\mathrm{OH}^{*}$ signal and simulated heat release rate distributions without acoustic forcing. Left : experiments. Middle : simulations with $\beta=0.5$. Right : simulations with $\beta=0.6$ 


\subsection{Scenario C: influence of the strain rate on the flame response}

In F-TACLES, the chemistry is tabulated from unstrained premixed laminar flames. It is assumed that the flame structure is not altered by the strain rate leading to a constant flame thickness. However, in the investigated configuration, the flame is subjected to large velocity fluctuations that stretch the flame up and down during a forcing cycle. To investigate this issue, the LES velocity fields are post-processed to estimate the resolved strain rate as follows:

$$
\tilde{S}=\sqrt{2\left(\widetilde{S_{i j}} \widetilde{S_{i j}}\right)} \text { where } \widetilde{S_{i j}}=\frac{1}{2}\left(\frac{\partial \tilde{u_{i}}}{\partial x_{j}}+\frac{\partial \tilde{u_{j}}}{\partial x_{i}}\right)
$$

Note that subgrid scale strain effects are not included in this expression and Eq. 10 only provides an estimate of the effective strain rate acting on the flame front elements. An instantaneous view of $\widetilde{S}$ is shown at the left in Fig. 19 for an excitation at $f=350 \mathrm{~Hz}$ and $u^{\prime} / \bar{u}=0.09$. The corresponding temperature field is plotted at the right in Fig. 19. As expected, the strain rate is high in the inner and outer shear layers of the swirling jet. As discussed in [30], simulations predict a thin residual reaction zone in the outer shear layer of the swirling jet, which is not observed in the $\mathrm{OH}^{*}$ light signal measured in the experiments. This region is also characterized by large heat losses [29]. This is highlighted by the temperature field in Fig. 19, where the burnt gases are cooled down to $800 \mathrm{~K}$ and the fresh gases from the swirling reactant stream meet the cooled burnt gases that are recirculating in the corner of the combustor.

The combined effects of heat losses and strain rates are not included in the LES model used in this work and may explain the differences observed between simulations and experiments $[27,28,53]$. One possibility would be to include these effects in the tabulated chemistry. One can mention the work presented in [25] or [54], where the effect of heat losses and strain rate is taken into account in the tabulation process. This raised however critical modeling issues out of the scope of the present study such as the estimation of unresolved strain rate in the LES. The following simplified approach is therefore preferred to analyze the results.

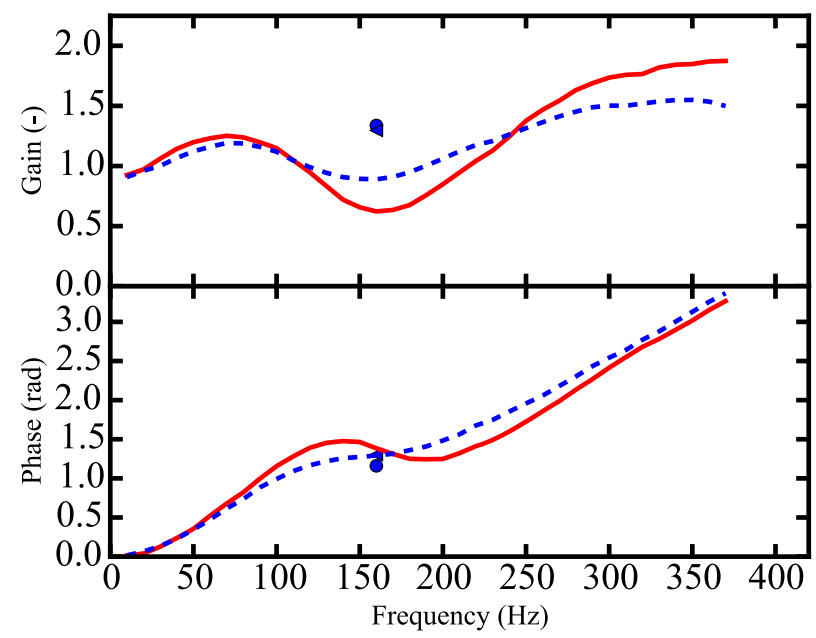

Fig. 18 FDF gain (top) and phase bottom. Experiments : $u^{\prime} / \bar{u}=0.09$ (red solid line), $u^{\prime} / \bar{u}=0.17$ (blue dashed lines). Simulations : $u^{\prime} / \bar{u}=0.17$ (blue items). Triangles: $\beta=0.5$. Circles: $\beta=0.6$ 
The following 1D numerical analysis is undertaken. A collection of 1D strained premixed flames is computed with the REGATH solver [42]. The simulated configuration consists in a $1 \mathrm{D}$ steady isobaric laminar premixed flame stabilized between two counter-flows. A $\mathrm{CH}_{4} / \mathrm{H}_{2}$ /air mixture is injected from one side at the equivalence ratio $\phi=0.70$ and temperature $T_{f}=298 \mathrm{~K}$. The opposite injector is fed with burnt gases of the same mixture for which a given enthalpy defect $\Delta h$ is applied. For that purpose, the equilibrium composition of the burnt gases at an imposed temperature is computed first for the $\mathrm{CH}_{4} / \mathrm{H}_{2}$ /air mixture composition injected in the system. The imposed burnt gas temperature is then varied between the adiabatic flame temperature reached in the absence of heat losses and the minimum temperature set here to $800 \mathrm{~K}$ approximately. This latter temperature roughly corresponds to the temperature of the burnt gases in the coldest zones of the combustor found in Fig. 19. The critical strain rate leading to flame quenching is then extracted from the 1D simulations as the strain rate for which the laminar flame speed of the non-adiabatic strained flame drops below $5 \mathrm{~cm} . \mathrm{s}^{-1}$. The results are plotted in Fig. 20 as a black line, delimiting two zones. For a given enthalpy loss, combustion persists below a specific strain rate threshold level. Increasing the strain rate beyond this value results in flame extinction.

It is worth examining the LES calculations and see where the results are located in this diagram. The cases FT-160-09, FT-160-17, FT-350-09 are selected. Resolved (or filtered) value of the strain rate is picked in the LES calculations at $z=0 \mathrm{~mm}$ corresponding to the top of the central bluff-body and at the point of maximum heat release in the outer shear layer of the swirling jet. This particular location is selected because this is where the highest strain rates are observed in the outer shear layer of the jet in Fig. 21. The value of the enthalpy defect $\delta h$ needs also to be estimated and is taken here in the burnt gases at $x=10$ $\mathrm{mm}$ and $z=0 \mathrm{~mm}$. This region is again selected in the outer shear layer of the swirling jet at the same altitude $z$ as for the estimation of the strain rate, but in the cold burnt gases as shown in Fig. 20. The values for the strain rate and enthalpy defect are extracted from LES for twenty regularly distributed instants, each separated by $T / 10$, where $T=1 / f$ is the oscillation period of the forcing signal. This duration covers two full cycles of excitation. These data are then added to Fig. 20 for each case explored.

Figure 20 clearly shows that, even by neglecting unresolved (or subfilter) strain rate contribution, the flame should be quenched in the outer shear layer of the swirling jet at $z=0 \mathrm{~mm}$ for the three cases explored and all instants considered in the forcing cycle. The flame cannot sustain such levels of strain rate for these levels of enthalpy defect. One may also note that the values found lie quite far from the boundary of the quenched zone,
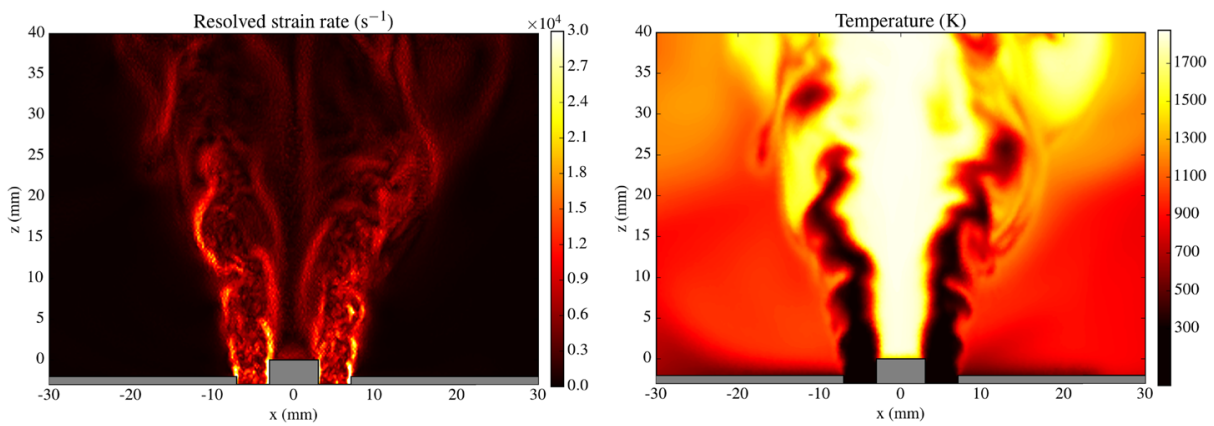

Fig. 19 Instantaneous LES resolved strain rate (left) and temperature (right) for the case FT-350-09 


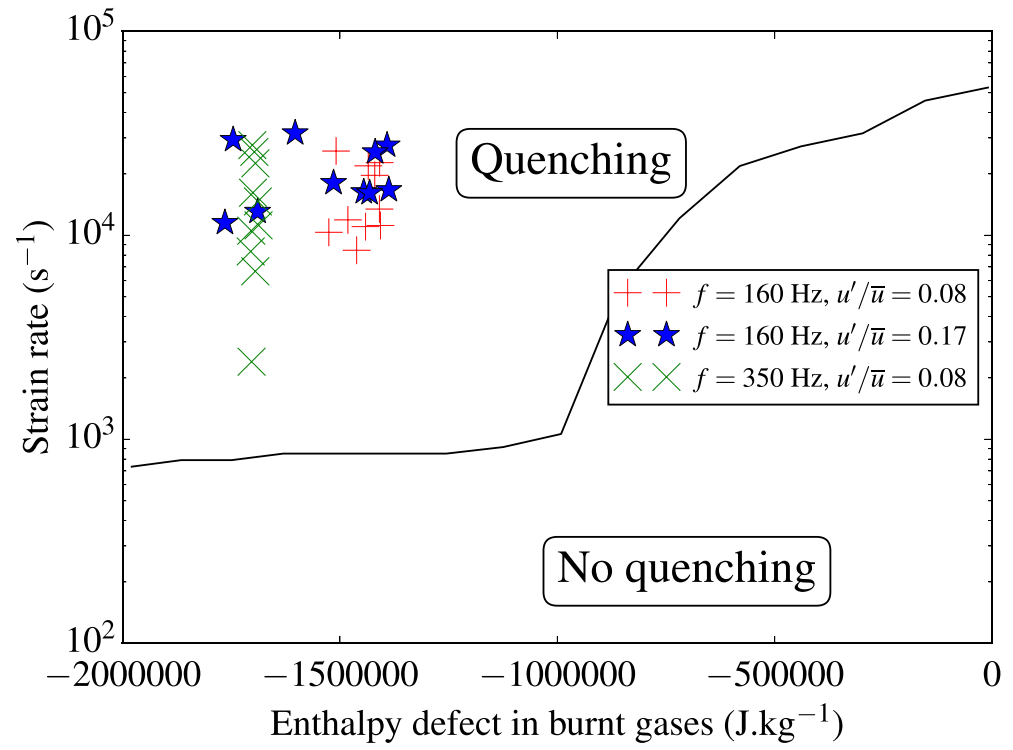

Fig. 20 Black line: critical strain rate as a function of the enthalpy defect delimiting the quenching and no quenching zones. Symbols: resolved strain rate at $z=0 \mathrm{~mm}$ and at the point of maximum heat release in the outer shear layer of the swirling jet as a function of the enthalpy defect in the burnt gases at $x=10 \mathrm{~mm}$ and $z=0 \mathrm{~mm}$

which has been calculated with a laminar counter-flow configuration. Slight variations of the enthalpy defect or strain rate estimates due to the region of interest in the outer reaction layer where these values are probed and effects of turbulence will thus not affect the conclusion. Therefore, the limitations of the combustion modeling may explain the remaining reaction zone in the outer shear layer of the swirling jet.

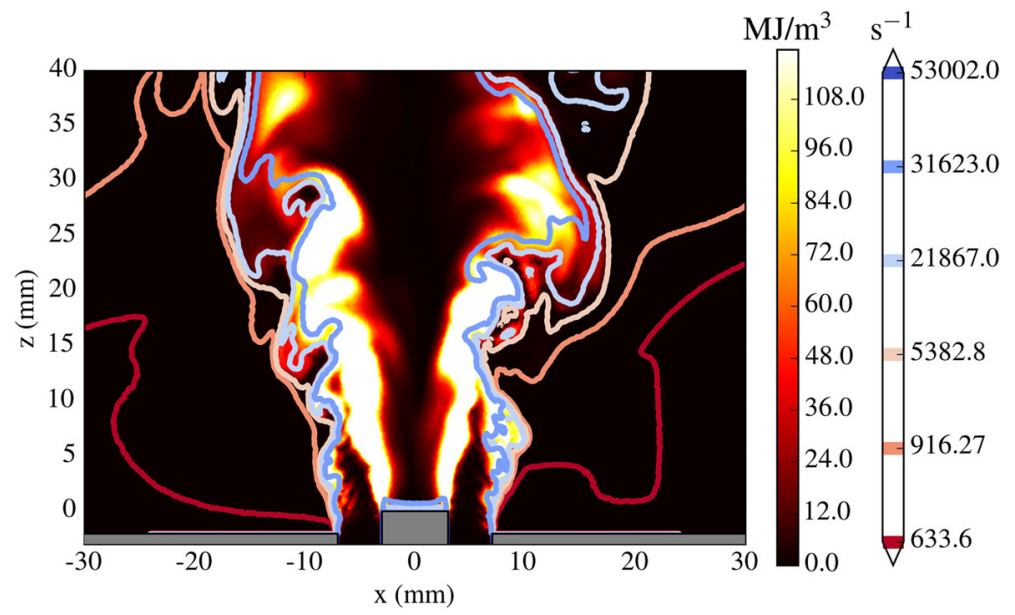

Fig. 21 Instantaneous heat release rate field for case FT-350-09 with superimposed isolines corresponding to the critical strain rate for a given enthalpy defect (extracted from Fig. 20) 
This is confirmed by a supplementary post-processing of the numerical results which demonstrates the combined effects of strain and enthalpy defect on the turbulent flame topology. By using laminar flame data from Fig. 20, isolines of the critical strain rate associated to a given enthalpy defect are indicated in Fig. 21, at one selected time instant of case FT350-09. This post-processing avoids the need to model unresolved strain rate contributions. Being superimposed to the field of heat released, these isolines evidence regions where the strain rate would lead to flame quenching. Even at moderate levels of strain rate (as low as $600 \mathrm{~s}^{-1}$ ), flame quenching by strain would occur primarily in the outer flame region. At the opposite, the effect of strain rate on the inner flame region is not significant, as the enthalpy defect in this region is small compared to the one in the outer recirculation zone.

The response of this additional reaction layer is considered in the computation of the FDF gain in the numerical results. The differences in flame response between $f=160$ and $350 \mathrm{~Hz}$ result in a variable additional gain due to the response of the outer reaction zone to acoustic forcing. At the forcing frequency $f=160 \mathrm{~Hz}$ and $u^{\prime} / \bar{u}=0.16$, the flame envelope varies greatly in height over an excitation cycle (Fig. 9), the outer reaction zone changing accordingly. At $f=350 \mathrm{~Hz}$ and $u^{\prime} / \bar{u}=0.16$, the flame envelope is less altered by the forcing with a weakly evolving outer reaction zone. Therefore the additional FDF gain is more noticeable at $f=160 \mathrm{~Hz}$ than at $f=350 \mathrm{~Hz}$. The FDF data in Fig. 5 corroborate this scenario.

\section{Conclusion}

Comparisons between experiments and numerical simulations for the FDF of a swirlstabilized premixed flame have been carried out. The FDF phase shift between velocity and heat release rate fluctuations integrated over the flame volume is fairly well reproduced by the simulations for the two modulation levels $u^{\prime} / \bar{u}=0.09$ and 0.17 examined. However, differences appear for the FDF gain at both forcing levels. Detailed examinations of the flame response at two forcing frequencies $f=160 \mathrm{~Hz}$ and $f=350 \mathrm{~Hz}$ indicate differences in the way the volumetric heat release is distributed over the flame raising questions regarding the modeling choices. An analysis of the dynamics of the turbulent flame brush envelope to the acoustic excitation reveals a complex response that depends on the forcing conditions. The current turbulent combustion formalism (F-TACLES) makes the assumption that the flame stays in the regime of unstretched flamelets with constant subgrid flame wrinkling parameters. These two hypotheses need to be reconsidered for the pulsated flows analyzed in this study. Several scenarii are explored and show that the main issue that should be taken into account in the numerical modeling approach is the combined effects of strain rate and enthalpy defect on flame structure.

Acknowledgements Vincent Moureau and Ghislain Lartigue from CORIA are acknowledged for providing the YALES2 flow solver through the SUCCESS scientific group.

Funding Information This work was performed using HPC resources from GENCI-IDRIS (Grants 2015x20152b0164 and 2016-x2016b0164). This work was supported by the ANR-10-EESI-0005 Grant of the French Ministry of Research.

\section{Compliance with Ethical Standards}

Conflict of interests The authors declare that they have no conflict of interest. 


\section{References}

1. Poinsot, T.: Proc. Combust. Inst. 36, 1 (2017)

2. Komarek, T., Polifke, W.: J. Eng. Gas Turbines Power 132(6), 061503 (2010)

3. Palies, P., Durox, D., Schuller, T., Candel, S.: Combust. Flame 157(9), 1698 (2010)

4. Kim, K.T., Hochgreb, S.: Combust. Flame 158(12), 2482 (2011)

5. Cosic, B., Terhaar, S., Moeck, J., Paschereit, C.: Combust. Flame 162, 1046 (2015)

6. Noiray, N., Durox, D., Schuller, T., Candel, S.: J. Fluid Mech. 615, 139 (2008)

7. Tay-Wo-Chong, L., Bomberg, S., Ulhaq, A., Komarek, T., Polifke, W.: J. Eng. Gas Turbines Power 134(2), 021502 (2012)

8. Iudiciani, P., Duwig, C.: Flow Turbul. Combust. 86, 639 (2011)

9. Palies, P., Schuller, T., Durox, D., Gicquel, L., Candel, S.: Phys. Fluids 23(037101), 15 (2011)

10. Krediet, H., Beck, C., Krebs, W., Schimek, S., Paschereit, C., Kok, J.: Combust. Sci. Technol. 184(7-8), 888 (2012)

11. Tay-Wo-Chong, L., Polifke, W.: J. Eng. Gas Turbines Power 135(2), 021502 (2013)

12. Hermeth, S., Staffelbach, G., Gicquel, L., Anisimov, V., Cirigliano, C., Poinsot, T.: Combust. Flame 161(1), 184 (2014)

13. Bauerheim, M., Staffelbach, G., Worth, N., Dawson, J., Gicquel, L., Poinsot, T.: Proc. Combust. Inst. 35, 3355 (2015)

14. Han, X., Morgans, A.S.: Combust. Flame 162, 1778 (2015)

15. Huang, Y., Yang, V.: Prog. Energy Combust. Sci. 35(4), 293 (2009)

16. Candel, S., Durox, D., Schuller, T., Bourgouin, J.F., Moeck, J.P.: Annu. Rev. Fluid Mech. 46, 147 (2014)

17. Poinsot, T., Veynante, D.: Theoretical and Numerical Combustion (RT Edwards, Inc.) (2005)

18. Pitsch, H.: Rev, Annu. Fluid Mech. 38, 453 (2006)

19. Fiorina, B., Mercier, R., Kuenne, G., Ketelheun, A., Avdić, A., Janicka, J., Geyer, D., Dreizler, A., Alenius, E., Duwig, C., et al.: Combust. Flame 162(11), 4264 (2015)

20. Tay Wo Chong, L., Komarek, T., Kaess, R., Föller, S., Polifke, W.: In: Proceedings of the ASME Turbo Expo 2010, ed. by ASME (ASME Turbo Expo 2010 : Power for Land, Sea and Air, June 14-18, 2010, Glasgow, UK) (2010). GT2010-22769

21. Tay Wo Chong, L., Polifke, W.: J. Eng. Gas Turbines Power 135 (9 pages), 021502 (2013)

22. AVBP website. www.cerfacs.fr/avbp7x/

23. Legier, J.P., Poinsot, T., Veynante, D.: In: Proceedings of the summer program, pp. 157-168 (2000)

24. Komarek, T., Chong, L.T.W., Zellhuber, M., Huber, A., Polifke, W.: In: Int. Conf. on jets, wakes and separated flows, Technical University of Berlin (2008)

25. Chong, L.T.W., Komarek, T., Zellhuber, M., Lenz, J., Hirsch, C., Polifke, W.: In: Proceedings of European Comb. Meeting (2009)

26. Keppeler, R., Pfitzner, M., Chong, L.T.W., Komarek, T., Polifke, W.: In: Proceedings of ASME Turbo Expo, pp. 457-467 (2012)

27. Tay Wo Chong, L., Komarek, T., Zellhuber, M., Hirsch, C., Polifke, W.: Flow Turbul. Combust. 97, 263 (2016)

28. Tay Wo Chong, L., Scarpato, A., Polifke, W.: In: GT2017-63357 in Proceedings of ASME Turbo Expo 2017: Turbomachinery Technical Conference and Exposition GT2017 June 26-30, 2017, Charlotte, NC, USA (2017)

29. Guiberti, T.F., Durox, D., Scouflaire, P., Schuller, T.: Proc. Combust. Inst. 35(2), 1385 (2015)

30. Mercier, R., Guiberti, T., Chatelier, A., Durox, D., Gicquel, O., Darabiha, N., Schuller, T., Fiorina, B.: Combust. Flame 171, 42 (2016)

31. Code_Saturne website. code-saturne.org/cms/

32. Boger, M., Veynante, D., Boughanem, H., Trouvé, A.: Symp. Combust. 27(1), 917 (1998)

33. Fureby, C.: Proc. Combust. Inst. 30(1), 593 (2005)

34. Balachandran, R., Ayoola, B., Kaminski, C., Dowling, A., Mastorakos, E.: Combust. and Flame 143(1), 37 (2005)

35. Kedia, K., Altay, H., Ghoniem, A.: Proc. Combust. Inst. 33, 1113 (2011)

36. Mejia, D., Selle, L., Bazile, R., Poinsot, T.: Proc. Combust. Inst. 35, 3201 (2015)

37. Kedia, K., Ghoniem, A.: Proc. Combust. Inst. 35, 1065 (2015)

38. Moureau, V., Domingo, P., Vervisch, L.: Comptes Rendus Mé,canique 339(2), 141 (2011) 
39. Nicoud, F., Toda, H.B., Cabrit, O., Bose, S., Lee, J.: Phys. Fluids 23(8), 085106 (2011)

40. Fiorina, B., Vicquelin, R., Auzillon, P., Darabiha, N., Gicquel, O., Veynante, D.: Combust. Flame 157(3), 465 (2010)

41. Mercier, R., Auzillon, P., Moureau, V., Darabiha, N., Gicquel, O., Veynante, D., Fiorina, B.: Flow Turbul. Combust. 93(2), 349 (2014)

42. Franzelli, B., Fiorina, B., Darabiha, N.: Proc. Combust. Inst. 34(1), 1659 (2013)

43. Lindstedt, P.: 12 Month progress report 1, tech. rep. tr-96 009. Tech. rep. Brite Euram Program Project BRPR950056 (1997)

44. Charlette, F., Meneveau, C., Veynante, D.: Combust. Flame 131(1), 159 (2002)

45. Palies, P., Durox, D., Schuller, T., Candel, S.: J. Fluid Mech. 672, 545 (2011)

46. Durox, D., Schuller, T., Candel, S.: Proc. Combust. Inst. 30, 1717 (2005)

47. Oberleithner, K., Schmiek, S., Paschereit, C.: Combust. Flame 162, 86 (2015)

48. Schuller, T., Durox, D., Candel, S.: Combust. Flame 134, 21 (2003)

49. Preetham, Hemchandra, S., Lieuwen, T.: J. Propuls. Power 24(6), 1390 (2008)

50. Bunce, N.A., Quay, B.D., Santavicca, D.A.: J. Eng. Gas Turbines Power 136(2), 021503 (2013)

51. Armitage, C., Balachandran, R., Mastorakos, E., Cant, R.: Combust. Flame 146(3), 419 (2006)

52. Hall, J.M., Petersen, E.L.: In. J. Chem. Kinet. 38(12), 714 (2006)

53. Klarmann, N., Sattelmayer, T., Weiqung, G., Magni, F.: AIAA paper (2016-2120) (2016)

54. Breda, P., Zips, J., Pfitzner, M.: In: Proceedings of the 3rd World congress on momentum, heat and mass transfer (MHMT'18) (2018)

\section{Affiliations}

\section{Adrien Chatelier ${ }^{1,2}$ (1) . Thibault Guiberti ${ }^{1,3} \cdot$ Renaud Mercier ${ }^{4}$. Nicolas Bertier ${ }^{2} \cdot$ Benoît Fiorina ${ }^{1}$. Thierry Schuller ${ }^{1,5}$}

1 Laboratoire EM2C, CNRS, CentraleSupélec, Université Paris-Saclay, 3, rue Joliot Curie, 91192 Gif-surYvette Cedex, France

2 ONERA, BP72 - 29 Avenue de la Division Leclerc, 92322 Châtillon Cedex, France

3 Clean Combustion Research Center, KAUST, Thuwal 23955, Saudi Arabia

4 SAFRAN Tech, Rue des Jeunes Bois, Châteaufort - CS 80112, 78772 Magny-les-Hameaux, France

5 Institut de Mécanique des Fluides de Toulouse, IMFT, Université de Toulouse, CNRS, Toulouse, France 